Document downloaded from:

http://hdl.handle.net/10251/103255

This paper must be cited as:

Elena Sánchez García; Balaguer-Beser, A.; Pardo Pascual, JE. (2017). C-Pro: A coastal projector monitoring system using terrestrial photogrammetry with a geometric horizon constraint. ISPRS Journal of Photogrammetry and Remote Sensing. 128:255-273. doi:10.1016/j.isprsjprs.2017.03.023

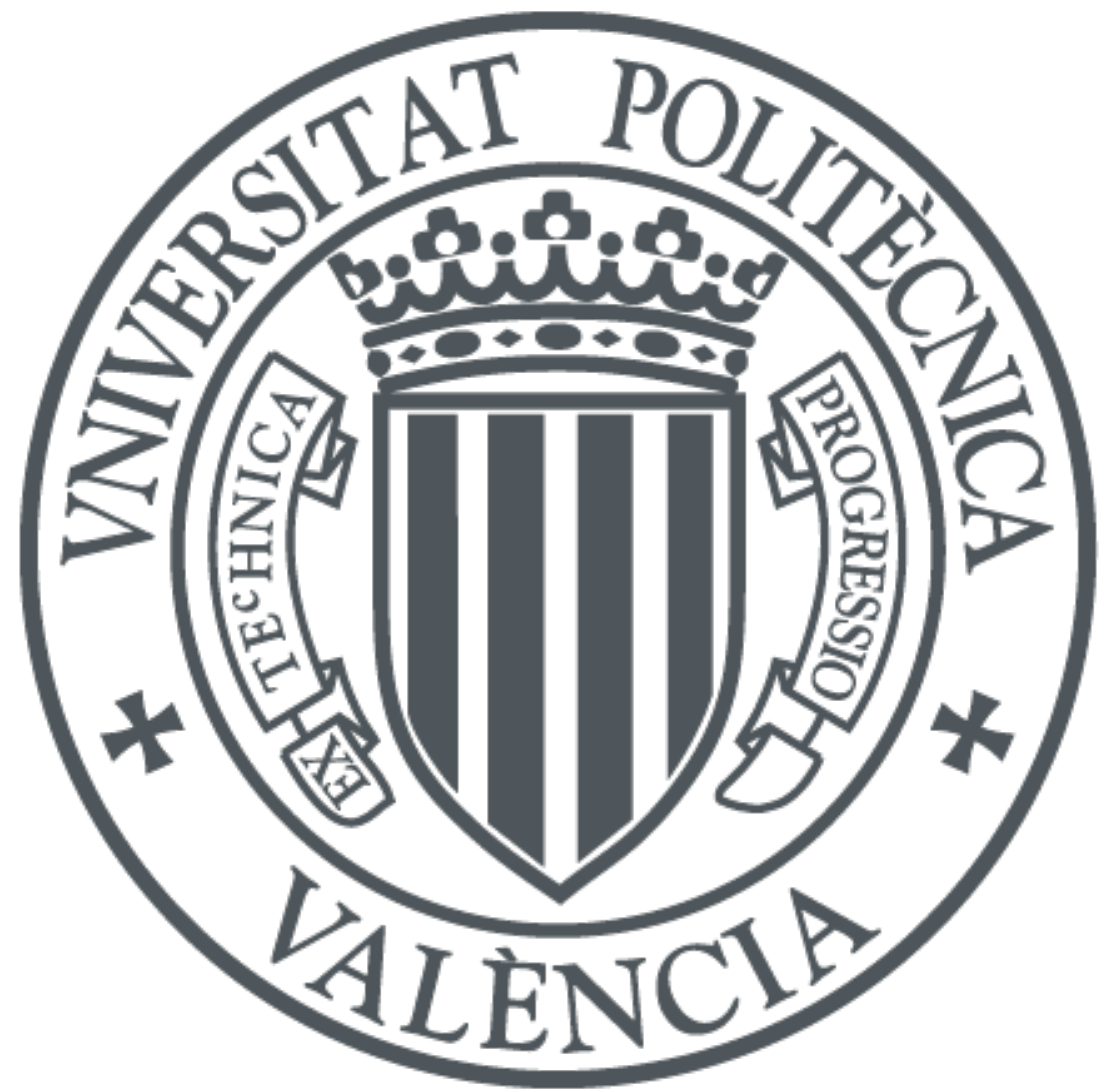

The final publication is available at

https://doi.org/10.1016/j.isprsjprs.2017.03.023

Copyright Elsevier

Additional Information 


\title{
C-Pro: A coastal projector monitoring system using terrestrial photogrammetry with a geometric horizon constraint
}

\author{
E. Sánchez-García ${ }^{\mathrm{a}}$, A. Balaguer-Beser ${ }^{\mathrm{a}, \mathrm{b}}$, J.E. Pardo-Pascual a \\ ${ }^{\text {a }}$ Geo-Environmental Cartography and Remote Sensing Group, Department of Cartographic Engineering, \\ Geodesy and Photogrammetry, Universitat Politècnica de València, Camí de Vera, s/n 46022, Valencia, Spain \\ ${ }^{\mathrm{b}}$ Department of Applied Mathematics, Universitat Politècnica de València, Camí de Vera, s/n 46022, \\ Valencia, Spain \\ * Corresponding author. E-mail address: elena.sanchez.upv@gmail.com
}

\section{ABSTRACT:}

This paper describes a methodological protocol to project a terrestrial photograph of a coastal area - or whatever indicator is contained on it - in a georeferenced plane taking advantage of the terrestrial horizon as a geometric key. This feature, which appears in many beach photos, helps in camera repositioning and as a constraint in collinearity adjustment. This procedure is implemented in a tool called Coastal Projector (C-Pro) that is based on Matlab and adapts its methodology in accordance with the input data and the available parameters of the acquisition system. The method is tested in three coastal areas to assess the influence that the horizon constraint presents in the results. The proposed methodology increases the reliability and efficient use of existing recreational cameras (with non-optimal requirements, unknown image calibration, and at elevations lower than $7 \mathrm{~m}$ ) to provide quantitative coastal data.

KEY WORDS: Beach monitoring, recreational cameras, coastal management, video-imaging system

\section{Introduction}

A proper management and planning of coastal areas is governed by an accurate understanding of these fragile and dynamic environments at different spatial and temporal scales. Modelling the coastline response to the effect of waves and sea level variation, especially in significantly unstable coasts such as sedimentary beaches, enables the evaluation of coastal retreat and coastline migration on large temporal scales. However, the complexity of the phenomena and processes that interact on the land-sea interface, makes this a deeply dynamic space in its form and arrangement (Boak and Turner, 2005). It is necessary to distinguish between oscillatory short-term effects and other long-term changes - and so monitoring changes at different temporal scales is helpful in a decision-making process involving environmental values and socioeconomic interests.

The spatial resolution and high temporal frequency achieved by terrestrial photogrammetric techniques have overcome the accuracy of other techniques in the field of monitoring. Techniques such as Airborne Light Detection and Ranging (LiDAR), Terrestrial Laser Scanner (TLS) and Global Positioning Systems in RealTime Kinematic (RTK-GPS) define the shoreline and model the beach area with accuracy and reliability despite tedious fieldwork and costs. However, the high periodicity required to monitor dynamics in natural spaces is causing these techniques to be set aside. Conversely, remote sensing techniques are being used to establish and quantify erosion or accretion rates on beaches and the results are sufficiently accurate - in the order of several meters - to help in our understanding and prediction of long-term worldwide coastal evolution (Almonacid-Caballer et al., 2016). Nevertheless, its potential is reduced for local studies and shortterm changes where video monitoring systems are consolidated as the current benchmark. 
Terrestrial photogrammetric systems enable a systematic and continuous recording of the different actions that take place in a specific coastal area. For instance, the local and rapid changes that occur during storms. Some institutions have realized the need to establish a proper and integrated coastal zone management and various video monitoring systems have been installed. The Argus system was the first developed for coastal research (Holman et al., 1993) and was validated and widely used worldwide (Holman and Stanley, 2007). Following the same principles, other coastal imaging systems were implemented. Archetti et al. (2008) made a comparative study of four fixed-camera systems: Erdman (1998); Kosta (2006); Horus (2007); and Beachkeeper (Brignone et al., 2012). Moreover, various works (Jiménez et al., 2007; Davidson et al., 2007; Aarninkhof et al., 2003) widely recognize the success of video systems for coastal research and shoreline monitoring through video-derived coastal indicators. Recent developments have emerged that access the digital image data from non-expert systems and regardless of the camera technology (e.g.,Taborda and Silva, 2012; Kim et al., 2013).

Existing coastal imaging systems are ready focused and dedicated for a specific application and this leads to some economic and positioning limitations. The measurement of shorelines, sand bars, beach widths, and many other indicators is easy to accomplish using fixed cameras covering wide fields of view and located on high elevation beach-front buildings. However, these optimal requirements are unusual on most beaches around the world and so other approaches are being investigated.

Many recreational video-cameras are currently operating on the coastline and sending considerable data over the internet - as well as a small number of systems designed by coastal managers in specific areas to control storm events. Most of this data is captured by Surfcam stations whose main qualitative objective is to observe breaking waves. As expected, the camera requirements are not optimal for quantitative measurements as they are low-angle and single cameras mounted on low beachfront buildings and pointing nearly horizontal toward the waves. Making the most of all the data from such shoreline monitoring cameras is the challenge tackled in this paper and complementing other papers (Bracs et al., 2015) where the potential of Surfcam data has already been proven through applying various solutions.

We propose a rigorous methodology - implemented in a coastal projector tool known as C-Pro - that overcomes the photogrammetric difficulties and non-optimal conditions that are sometimes found in beach photographs. The main goal is to use the terrestrial horizon as a photogrammetric constraint included in the collinearity system to achieve a precise repositioning of the camera (Sánchez-García et al., 2015b). Van Den Heuvel (1998) already advanced the benefits of using geometric constraints for object reconstruction. When using a simple non-metric camera looking horizontally towards the coastline and from any elevation - even from the ground where there is no other option - the horizon constraint helps the image spatial resection system to converge on a precise solution that is valid for coastal monitoring. Moreover, because of the field of view, most of the photos only show sand and water, and this makes it difficult to acquire ground control points (GCP) with a suitable distribution to transform image information into real world coordinates. Reducing the number of initial unknown parameters by adding horizon equations (Oreifej et al., 2011) would be a great advantage in providing stability to the mathematical system.

Some works that use Surfcam online streaming images for measuring wave runup and intertidal beach topography (Andriolo et al., 2016) are already taking advantage of C-Pro rectification methodology as the horizon constraint is the key to achieving image calibration and a precise repositioning of the camera.

In this paper, Section 2 describes the different methodological steps depending on the number of parameters initially known, access to camera calibration, knowledge of the initial location of the camera, or number of available GCPs. Section 3 shows the results obtained after camera repositioning and image rectification processes and shows the considerable advantages of incorporating the horizon constraint. Finally, Appendix A 
presents the mathematical formulas necessary to incorporate horizon information in a monitoring system using terrestrial photogrammetry.

\section{Methodology}

\subsection{A photogrammetric system}

The analytical method consists of three main processes: calibration and image correction; repositioning of the camera; and image rectification. The followed protocol establishes a strong and rigorous geometric connection between both terrestrial and image spaces with the implementation of the horizon constraint in the collinearity system (described in Appendix A). Moreover, the tool will compute the adjustment adapting to different situations depending on the number of initially known and unknown external and internal orientation parameters.

\subsubsection{Camera calibration and image correction}

In photogrammetry, the extraction of metric information requires a precise knowledge of the internal orientation parameters (IOP) - principal point coordinates $\mathrm{o}=\left(x_{0}, y_{0}\right)$ and focal length $(\mathrm{f})$ - and the distortion coefficients of the non-metric camera lens (assuming rectangular pixels skew factor is generally negligible). A camera acquires images composed of pixels where each pixel captures light traveling along the projection of a $3 \mathrm{D}$ ray. The projection rays in principle can be placed arbitrarily assuming the absence of a functional relationship between the projection rays and the pixels directed by the intrinsic parameters. Thus, the calibration is described in accordance with the coordinates of these rays (given in the local coordinate system) and the correspondence between the rays and pixels. After such calibration, each ray of the bundle passes correctly through the optical center.

In the present work, as we had access to the cameras, an a-priori laboratory study of the acquisition system itself was made. It is known that self-calibration can improve the accuracy of non-metric cameras (Chandler et al., 2005). This shows the potential that cheap cameras have for measuring surfaces when the lens model has been considered and a correct calibration of the intrinsic camera parameters has been made.

The calibration involves applying the Matlab camera calibration toolbox of Bouguet (2015). This calibration tool works with a series of images on a pattern like a checkerboard with the camera focused to infinity and taking the photos from different points of view and changing orientation and position. The IOP are estimated by an initial approach linearizing the equations and a least squares adjustment. These parameters are generally invariant and unique for each camera under similar conditions (Holland et al., 1997). Removing the induced effects of these intrinsic camera parameters, the image is corrected and undistorted by the empirical inverse model for compensating lens distortions proposed by Heikkila and Silvén, 1997. After the image correction, a correct geometric relation between the image and terrain systems will exist and the center of the undistorted image will coincide with the center of the original image - and will be consistent with the formulas shown in the following sections.

\subsubsection{Camera repositioning}

The process of determining the orientation parameters is understood as spatial resection and is considered as a particularization of a photogrammetric triangulation for a simple image. The six external orientation parameters (EOP) recreates the moment in which a photo is taken and defines the object coordinates of the camera center $\left\{\mathrm{X}_{C_{C}}, \mathrm{Y}_{C_{C}}, \mathrm{Z}_{C_{C}}\right\}$, and its orientation angles $\{\omega, \varphi, \kappa\}$. Thus, it is necessary to establish a strong 
and effective relation between the terrain and the image spaces before using the photos for photogrammetric purposes.

The protocol carried out in the present work to calculate the orientation parameters follows one of two methodologies - depending on the number of available GCPs (three being the minimum).

\subsubsection{Direct Linear Transformation}

Direct linear transformation (DLT) (Abdel-Aziz and Karara, 1971) is the most widely user linear camera calibration method because of its simplicity (Bacakoglu and Kamel, 1997). DLT does not require initial knowledge of the approximate orientation parameters because these are implicit in the 11 transformation parameters. DLT theoretically adapts better to specific tasks, especially to close range photogrammetry which differs from our goals in coastal areas -. However, inside our protocol, DLT usefully provides the initial approximated values for those unknown EOP in which the collinearity least squares fitting needs input data to start. A great benefit of the DLT method is its linear quality regarding numerical problems that could appear and we must be careful when the control points are coplanar because then the 11 transformation parameters cease to be independent.

One control point with known terrain coordinates generates two linear equations which are expressed by the following system of linear equations:

$$
\left(\begin{array}{lllllllll}
X & Y & Z & 1 & 0 & 0 & 0 & 0 & -x X-x Y-x Z \\
0 & 0 & 0 & 0 & X & Y & Z & 1-y & -y X-y Y-y Z
\end{array}\right) L=\left(\begin{array}{l}
x \\
y
\end{array}\right) \rightarrow A L=\left(\begin{array}{l}
x \\
y
\end{array}\right)
$$

being $L=\left(L_{1}, L_{2}, L_{3}, L_{4}, L_{5}, L_{6}, L_{7}, L_{8}, L_{9}, L_{10}, L_{11}\right)^{T}$. An overdetermined set of linear equations: $M L=N$ is obtained by applying (1) for a minimum of six GCPs. We can then obtain L using the least square method.

The current relation between these 11 DLT parameters, $L$, and the other 9 (internal and external camera parameters) of the collinearity equations is well-known (Seedahmed and Habib, 2002). If it is possible to establish the internal parameters as a result of the camera calibration, or if the camera position is available, then it would be preferable to use these. Moreover, when the horizon appears in the photo, the values obtained by DLT will not be used as initials, because we would prefer those obtained by (A.26) and (A.27) - described at the end of appendix A.

\subsubsection{Refinement process by collinearity}

To guarantee a strong relationship between terrain and image spaces, an iterative adjustment system must be carried out to obtain the parameters that recreate the time of the shoot as faithfully as possible. This point of the mathematical process is where it is necessary to introduce a geometric constraint to add methodological rigor. Photographs of a beach area usually present homogeneous characteristics that hinder a proper distribution of the GCPs. However, the horizon curve is an essential strategic element. In this locus, all vanishing points of the image converge and therefore it acts as if we had a set of control points at infinity. Appendix A describes the details of a mathematical procedure for having characterized the horizon, relating it to the EOP by means of (A.22). Our methodological protocol proposes the inclusion of these novel constraints in the adjustment to obtain a much more accurate solution.

All iterative adjustment starts from an initial approximate solution of all the parameters. The prior values for the internal parameters can correspond with those obtained by DLT, or by the camera calibration process. Depending on the reliability of these initial parameters, the collinearity will then be resolved and so free all 
the parameters if they are from DLT, or freeing only six because the IOP will remain fixed (providing they are produced after calibration and are accurate enough).

The initial camera position coordinates are obtained by the camera user, but in cases where this is not possible they are approximated by the DLT method. When the horizon appears in the image, initial orientation parameters, $\{\omega, \varphi, \kappa\}$, are computed by means of (A.26) and (A.27). In other cases, those values are also obtained by the DLT.

The resolution of the spatial resection by the collinearity condition is carried out developing the classical nonlinear equations of the central projection which relates the position of the GCP, $(X, Y, Z)$, in the object space of the position of its image point $(\mathrm{x}, \mathrm{y})$ in the image plane.

In this paper, we propose to include the geometric horizon constraint in the refinement adjustment, using the mathematical procedure described in Appendix A. Thus, we consider equations (A.22) and we define the following functions:

$$
\left.\begin{array}{rl}
\mathrm{F}_{\mathrm{H} \xi}(\omega, \varphi) & =\arccos (\cos (\varphi) \cos (\omega))-\xi \\
\mathrm{F}_{\mathrm{H} \psi}(\omega, \varphi) & =\arctan \left(\frac{-\sin (\varphi)}{\cos (\varphi) \sin (\omega)}\right)-\psi
\end{array}\right\}
$$

considering that $\psi$ and $\xi$ are defined constants, respectively, by means of (A.1)-(A.3) and (A.12), using the horizon information through the marked points $\mathrm{A}, \mathrm{B}$, and $\mathrm{C}$ in the image. Thus, we add to the collinearity system these two new linearized equations:

$$
\left(\begin{array}{c}
\mathrm{F}_{\mathrm{H} \xi} \\
\mathrm{F}_{\mathrm{H} \psi}
\end{array}\right)_{0}+\left(\begin{array}{ccccccccc}
\frac{\partial \mathrm{F}_{\mathrm{H} \xi}}{\partial \omega} & \frac{\partial \mathrm{F}_{\mathrm{H} \xi}}{\partial \varphi} & 0 & 0 & 0 & 0 & 0 & 0 & 0 \\
\frac{\partial \mathrm{F}_{\mathrm{H} \psi}}{\partial \omega} & \frac{\partial \mathrm{F}_{\mathrm{H} \psi}}{\partial \varphi} & 0 & 0 & 0 & 0 & 0 & 0 & 0
\end{array}\right)\left(\begin{array}{c}
d \omega \\
d \varphi \\
d \kappa \\
\frac{d \mathrm{X}_{C_{C}}}{d Y_{C_{C}}} \\
d \mathrm{Z}_{C_{C}} \\
d x_{0} \\
d y_{0} \\
d f
\end{array}\right)=\left(\begin{array}{l}
0 \\
0
\end{array}\right)
$$

If the IOP have been computed by calibration, the refinement process only aims to obtain the correction for the six EOP $\left\{d \omega, d \varphi, d \kappa, d \mathrm{X}_{C_{C}}, d Y_{C_{C}}, \mathrm{dZ}_{C_{C}}\right\}$. By applying collinearity with a minimum of three GCP, a system formed by those classical collinearity equations together with (3) will then be solved. However, if the correction of the three IOP $\left\{d x_{0}, d y_{0}, d f\right\}$ also needs to be computed, then the tool will solve collinearity for nine parameters requiring at least four GCP. This case occurs when the IOP come from DLT. The whole spatial resection system will be expressed as,

being:

$$
\mathrm{B}(\mathrm{dP})=\mathrm{K}
$$

$$
d P=\left(d \omega, d \varphi, d \kappa, d \mathrm{X}_{C_{C}}, d Y_{C_{C}}, \mathrm{dZ}_{C_{C}}\right)^{T} \text { or } d P=\left(d \omega, d \varphi, d \kappa, d \mathrm{x}_{C_{C}}, d Y_{C_{C}}, \mathrm{dZ}_{C_{C}}, d x_{0}, d y_{0}, d f\right)^{T}
$$

considering the appropriate number of GCP for each of the situations commented above. System (4) will be resolved by the weighted least square method whose solution gives us the parameter correction:

$$
d P=\left(B^{T} W B\right)^{-1} B^{T} W K
$$


where $W$ is the diagonal weight matrix. The weight assigned to the classical collinearity equations is the same for all, but can vary depending on the reliability associated by the user with each of the GCP. As equations (3) should act as a constraint to the fitting, the weights assigned to these equations in our methodology are much higher than those used for the equations related to GCP. We will study the influence of those weights in Section 4. As a consequence, an accurate determination of the initial points to form the horizon approximation is important. In the results section, some performances analyze how the weight of the horizon equations influence in the resection adjustment.

We will consider an iterative process solving each step of the system (4) considering the parameters computed with the correction of the above iteration. Thus, in (4) B and K are defined in the $k$-iteration $(k \geq 1)$ using the solution obtained in the $(k-1)$-iteration so:

$\left(\omega^{k}, \varphi^{k}, \kappa^{k}, \mathrm{X}_{C_{C}}{ }^{k}, Y_{C_{C}}{ }^{k}, \mathrm{Z}_{C_{C}}{ }^{k}, x_{0}{ }^{k}, y_{0}{ }^{k}, f^{k}\right)=$

$\left(\begin{array}{c}\omega^{k-1}+d \omega^{k-1}, \varphi^{k-1}+d \varphi^{k-1}, \kappa^{k-1}+d \kappa^{k-1}, \mathrm{X}_{C_{C}{ }^{k-1}+d \mathrm{X}_{C_{C}}{ }^{k-1}, Y_{C_{C}}{ }^{k-1}+d Y_{C_{C}}{ }^{k-1},} \\ \mathrm{Z}_{C_{C}}{ }^{k-1}+d \mathrm{Z}_{C_{C}}{ }^{k-1}, x_{0}{ }^{k-1}+d x_{0}{ }^{k-1}, y_{0}{ }^{k-1}+d y_{0}{ }^{k-1}, f^{k-1}+d f^{k-1}\end{array}\right)$

That process finishes when each of the correction parameters becomes insignificant (the established threshold is equal to $\left.10^{-10}\right)$.

To establish the convergence of the system, at the beginning of the $k$-iteration, $(k \geq 1)$, the C-Pro tool will color in green the calculated image coordinates of the GCP by computing $(\mathrm{x}, \mathrm{y})$ by means of the collinearity equations, considering the parameters obtained using (6) and the $(\mathrm{X}, \mathrm{Y})$ coordinates of each GCP. Moreover, the horizon line will be approximated considering:

$$
d_{\text {horizon }}^{k}=-f^{k} * \tan \left(\arccos \left(\cos \left(\varphi^{\mathrm{k}}\right) \cos \left(\omega^{\mathrm{k}}\right)\right)-\arccos \left(\frac{\mathrm{z}_{C_{C}}{ }^{{ }+0.42 * \frac{\left(D^{k}\right)^{2}}{R t}}}{D^{k}}\right)\right)
$$

where $D^{k}$ is defined by means of (A.10) considering $\mathrm{Z}_{C_{C}}{ }^{\mathrm{k}}$ defined by (6), and:

$$
\psi_{\text {calc }}^{\mathrm{k}}=\arctan \left(\frac{-\sin \left(\varphi^{\mathrm{k}}\right)}{\cos \left(\varphi^{\mathrm{k}}\right) \sin \left(\omega^{\mathrm{k}}\right)}\right)
$$

using the following equation:

$$
y=y_{0}^{k}+d_{\text {horizon }}^{k} \cos \left(\Psi_{\text {calc }}^{\mathrm{k}}\right)-\tan \left(\Psi_{\text {calc }}^{\mathrm{k}}\right)\left(x-x_{0}^{k}-d_{\text {horizon }}^{k} \sin \left(\psi_{\text {calc }}^{\mathrm{k}}\right)\right)
$$

Thus, the horizon line is recomputed in each iteration with the newest parameters until the adjustment process ends.

\subsubsection{Image rectification and data extraction}

Once the repositioning camera has been fruitfully achieved, the rectification process can be done. In our tool, the user can choose whether to project a piece of the image or just an element contained therein over a specific plane with a known $\mathrm{Z}_{\mathrm{T}}$ value. 
Choosing the first option, the tool detects the image limits in terrain coordinates and a georeferenced grid is created for a specific pixel size on the specific $\mathrm{Z}_{\mathrm{T}}$. Each pixel is then filled by an image intensity value through inverse mapping techniques and using the nearest neighbor interpolation method. In this case, the final product is a rectified georeferenced image (Tiff World File) used by standard GIS applications.

Nevertheless, by following the second option, the user can digitalize a specific feature of interest as a coastal indicator - such as an established shoreline, the landward edge, the foredune toe, the cliff top - or just upload a file with the image coordinates that the C-Pro tool is expected to project. This coordinate rectification is then either done by collinearity equations or by the DLT - both methods lead to the same solution. In the last case, we have to convert the camera's internal and external parameters to the characteristic's 11 transformation parameters of DLT which satisfy equations (1). From the DLT equations we can obtain the planimetric terrain coordinates $(\mathrm{X}, \mathrm{Y})$ projecting the image coordinates $(\mathrm{x}, \mathrm{y})$ of the indicator of interest on a specific $\mathrm{Z}_{\mathrm{T}}$, considering the next linear equation system (10).

$$
\left\{\begin{array}{c}
\left(L_{5}-L_{9} y\right) X+\left(L_{6}-L_{10} y\right) Y=\left(L_{11} y-L_{7}\right) Z_{T}+y-L_{8} \\
\left(L_{1}-L_{9} x\right) X+\left(L_{2}-L_{10} x\right) Y=\left(L_{11} x-L_{3}\right) Z_{T}+x-L_{4}
\end{array}\right.
$$

It is interesting to note the importance of the $\mathrm{Z}_{\mathrm{T}}$ projection value because only the points located at this same elevation will be projected at the correct place. The remainder points will be displaced unless we project the photography over a digital terrain model supporting each pixel of the image with its associate altitudinal value. In this paper the photographs are projected above the mean sea level $\left(Z_{T}=M S L\right)$ because the key image feature to be correctly georeferenced is the shoreline. However, in order to know the errors of the image rectification process, some terrain points have been projected over its associated altimetric coordinate (measured by RTK-GPS) and assessed its positional accuracy by solving (12).

\subsection{Practical implementation of C-Pro}

This section shows the main steps in the implementation of the C-Pro.

\section{Step 1: Calibration and image correction.}

1a) With calibrated IOP: If we have access to the cameras then we compute the IOP - principal point coordinates $\mathrm{O}=\left(x_{0}, y_{0}\right)$ and focal length (f) - by image calibration. With unknown IOP: In the other cases, having at least six GCPs, the system calculates an IOP estimation by direct linear transformation (DLT).

1b) The image is corrected and undistorted by the empirical inverse model for compensating lens distortion described in Bouguet (2015).

\section{Step 2: Repositioning of the camera through the spatial resection process.}

2a) Initial camera position $\left\{\mathrm{X}_{C_{C}}, Y_{C_{C}}, \mathrm{Z}_{C_{C}}\right\}$ is estimated by the user. However, when it is not possible, C-Pro will offer a first approximation of this through the DLT method (Section 2.1.2.1).

2b) With horizon constraint (with HC): If the horizon appears in the image then, angle $\psi$ is defined by means of (A.1) (case two points) or (A.2)-(A.3) (case three points). Moreover, $\xi$ is computed using (A.12) through the marked horizon points in the image and considering the focal length (f) obtained in step 1 and the camera center elevation $Z_{C_{C}}$ defined in $\left.2 a\right) .\{\omega, \varphi, \kappa\}$ are then computed by means of (A.26) and (A.27). 
Without horizon constraint (without HC): in cases where the horizon equation is not available, $\{\omega, \varphi, \kappa\}$ are estimated by the DLT.

2c) C-Pro calculates the final EOP through an iterative weighted least squares fitting (5) over the linearized collinearity equations (two equations for each GCP), which starts with the parameters defined in $2 \mathrm{~b}$ ) and finishes when each of the correction parameters become less than $10^{-10}$. The weight assigned to those equations is equal to 1 , but can vary depending on the reliability associated by the user to each of the GCP. Moreover, in the cases with horizon constraint, the linearized horizon constraints (3) are added with an associated weight of $10^{12}$ to solve the photo geometry. The system will be resolved and freeing all parameters if the IOP in step 1 proceeds from DLT (with unknown IOP), or freeing only six of them when the IOP remains fixed by considering that they are computed in step 1 (with calibrated IOP).

2d) To discover how the convergence of the system is progressing, C-Pro colors green (at the end of each iteration) the calculated image coordinates of the GCP by computing $(\mathrm{x}, \mathrm{y})$ by means of the collinearity equations and considering the parameters obtained using (6). Moreover, the horizon line is also colored in green considering equation (9).

\section{Step 3: Image rectification}

3a) To project a piece of the image on a specific plane with a known $Z_{T}$ value, a georeferenced grid is created and each pixel is filled with an image intensity value through inverse mapping techniques and using the nearest neighbor interpolation method.

3b) Collinearity equations or the DLT equations given in (10) will be used to project an element contained in the image on a plane with a constant $\mathrm{Z}_{\mathrm{T}}$ coordinate. In the case of shoreline rectification, the MSL value is used as $\mathrm{Z}_{\mathrm{T}}$.

\section{Testing of the horizon constraint and C-Pro software}

Assessing the protocol proposed in this paper, the following subsections show the benefits achieved by including the horizon constraint in the spatial resection process.

The analyses are carried out in the three coastal areas described in this section and working with specific photogrammetry conditions that must be solved. The tests are made with non-fixed cameras. Therefore, in the first step of C-Pro, the IOP of the acquisition system was obtained through image calibration (Bouguet, 2015). However, some of the results of this section will be obtained when considering the IOP as unknown to prove that C-Pro also works accurately in such cases.

\subsection{Data and study area}

The analyses are carried out in three coastal areas. Two are in the region of Valencia on the Mediterranean coast (Spain) and are long micro-tidal beaches (tide regime is less than $0.18 \mathrm{~m}$ ) with low and sandy coastlines and a wide shoal. Patacona beach and El Saler beach are popular with tourists and have suffered a marked erosion in recent decades due to sand retention by the jetties of the port of Valencia - north of El Saler beach - that interrupts the littoral drift (Sánchez-García et al., 2015a). The third study area is Magoito beach, located in Sintra, on the Atlantic coast of Portugal. This is a mesotidal beach (tide range between 2 and $3 \mathrm{~m}$ ) with a long stretch of golden sand dotted with numerous rocks and imposing cliffs that rise from the beach. 
A photogrammetric analysis in the study area was carried out by simple non-fixed cameras. In the Valencian beaches the images were taken with a digital single-lens reflex camera (SONY DSLR-A330) whereas a Mobotix MX-M12D camera was used at Magoito. The zoom lens is fixed to infinity and care was taken to ensure that the photos do not blur. Furthermore, to achieve the different assessments shown in this paper, it was necessary to ensure that the terrestrial horizon appears in the photo, at least partly, as well as a minimum of six non-coplanar GCPs that are well spread out and clearly and unequivocally displayed (Sánchez-García et al., 2016). These theoretical conditions hardly ever occur because of the homogeneous media found on beaches, where most of the photo shows water and sand. Moreover, because GCPs generally cover a very small part of the whole picture, infinite control points representing the terrestrial horizon and located far from the camera help resolve the photogrammetric problem.

Concerning RTK-GPS data, existing terrain points were measured accurately in several previous field campaigns and acted as GCPs by solving the geometry of the photo through the link between image and terrain systems - or only as checkpoints for assessing the solvency of that photogrammetric solution and image rectification. The stability of these points was ensured during all the evaluation process. The camera coordinates were also acquired by GPS to subsequently measure the error obtained in camera positioning. The planimetric coordinates (XY) and orthometric altitudes (Z) are accurate to less than $2 \mathrm{~cm}$ in planimetry and within $4 \mathrm{~cm}$ in altimetry (referenced respectively in the UTM projection - GRS80 - and the EGM08 geoid model).

Magoito beach is a study area with good characteristics for the usual photogrammetry requirements. It was possible to locate the camera in a high place - an elevation of $35.6 \mathrm{~m}$-, where a broad view of the beach area is seen and the horizon covers the entire width of the image (see Fig. 1). Moreover, during low tide it was possible to obtain 28 GPS points by taking advantage of some rocky elements that remained in sight. The distribution of these points through the target area - in this case generally centered in front of the camera will condition the significance of the positional error, as we will see in the results section when we must distinguish between both longitudinal and cross-shore components of error.

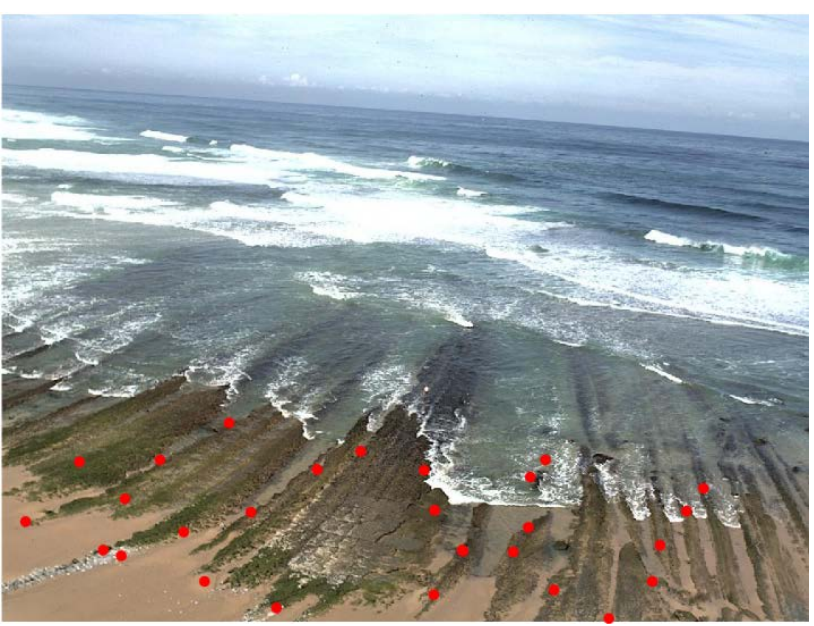

Fig. 1. Photo taken on Magoito beach at low tide. The GPS points (GCP and checkpoints) are shown in red.

Secondly, the coastal photos at El Saler beach were taken from two non-fixed camera positions at the top of a $43 \mathrm{~m}$ high building but separated from the shore by around $230 \mathrm{~m}$ (a park with protected coastal dunes being between the building and the coast). Consequently, the shoreline is partially hidden in the photos. We call the shots CS or CN depending on the camera location on the south or north side of the building from where three and four photographs were taken respectively each day. From a specific position, the photos were taken 
sequentially turning from north to south and numbering them by order. CN1 and CS1 photos capture almost the same target area but from another camera position. Moreover, depending on the photo orientation, the extent of horizon seen in the photos changes and we will analyze this in the results section to establish how this influences the horizon approximation formulas. Differences regarding the extent of the horizon are obvious in Fig. 2 depending on the existence of elements that hide it - such as the Port of Valencia in the northern part (CN1) and, to a lesser extent, the Cullera headland to the south (CS3). GPS points were measured along the entire area (about one kilometer long) and these points were included in photos that took advantage of fixed elements - outside the beach area - such as parking borders and pedestrian walkways.

The photogrammetric field campaigns were made during two days, 25 May and 17 June 2016, when the water/land border was measured (using RTK-GPS to record automatic coordinates every second). The availability of this data enables us to make comparisons with other digitized shorelines for the resulting and rectified photos and assess accuracy.

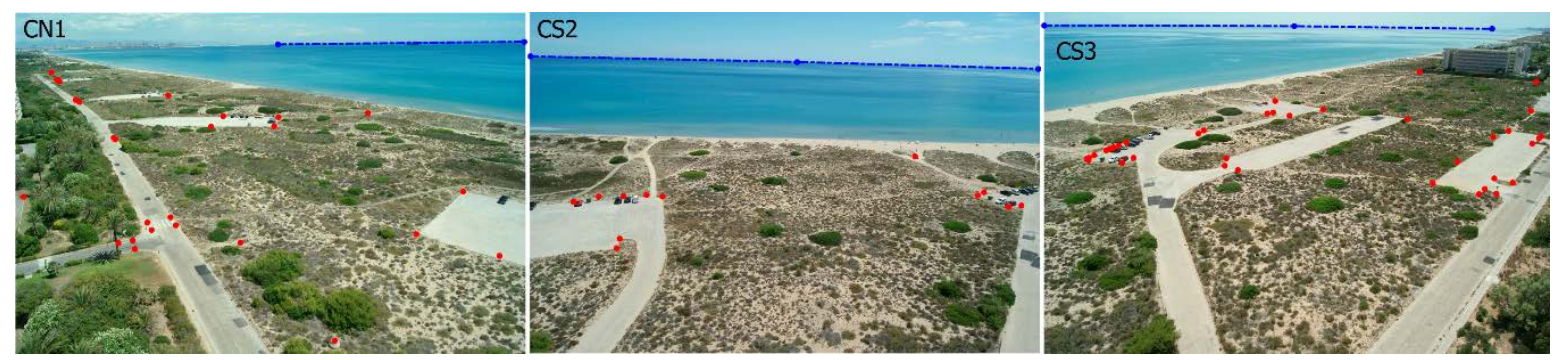

Fig. 2. Three examples of photos at El Saler beach taken from different camera position on 17 June 2016; the first photo from the north camera (NC) and the other two from the south camera (CS). Distribution of the GPS points in the area is dotted in red and the horizon line is shown in blue. CN1 is an example of where the horizon formulas are obtained by two points, while CS2 and CS3 are obtained by three points.

Finally, the third study area is Patacona beach where an additional longshore assessment was made on 22 September 2015. The photogrammetric procedure was performed twice - from same camera position but at different elevations - pointing north and south (changing the camera position). Taking advantage of a gangway near the shore, the photos were taken using a tripod $\left(Z_{C_{C}}=4.7 \mathrm{~m}\right)$ to hold the spatial resection of the camera. However, photos were then carefully taken from a handheld camera at the top of a stepladder $\left(Z_{C_{C}}=6.8 \mathrm{~m}\right)$. Consequently, we have different solutions of the camera spatial resection for each image. Given the absence of fixed elements in the beach area, several surveying rods were used as GCPs (black points on the map and photos in Fig. 3), producing a maximum of six and nine GCPs respectively for the northern and southern photos.

The main goal of the experiment carried out in this area was to understand the functioning of our methodology when camera elevation is reduced. Moreover, to measure the longshore error after image rectification, a field campaign took a set of photos coincident in time with the data acquisition every $20 \mathrm{~m}$ for two or three GPS trackers. These devices were separated some $2 \mathrm{~m}$ apart and measured moving checkpoints for a distance of $280 \mathrm{~m}$ from the camera. We had to establish a distance limit because of the subsequent difficulties in the detection of those checkpoints in the image. Fig. 3 exemplifies the experiment performed showing a photo of the north beach and of the south beach where respectively the positions (checkpoints) of three and two GPS trackers are detected. We can also see that the horizon appears in less than half the image and so the horizon approximation formula uses two points. 


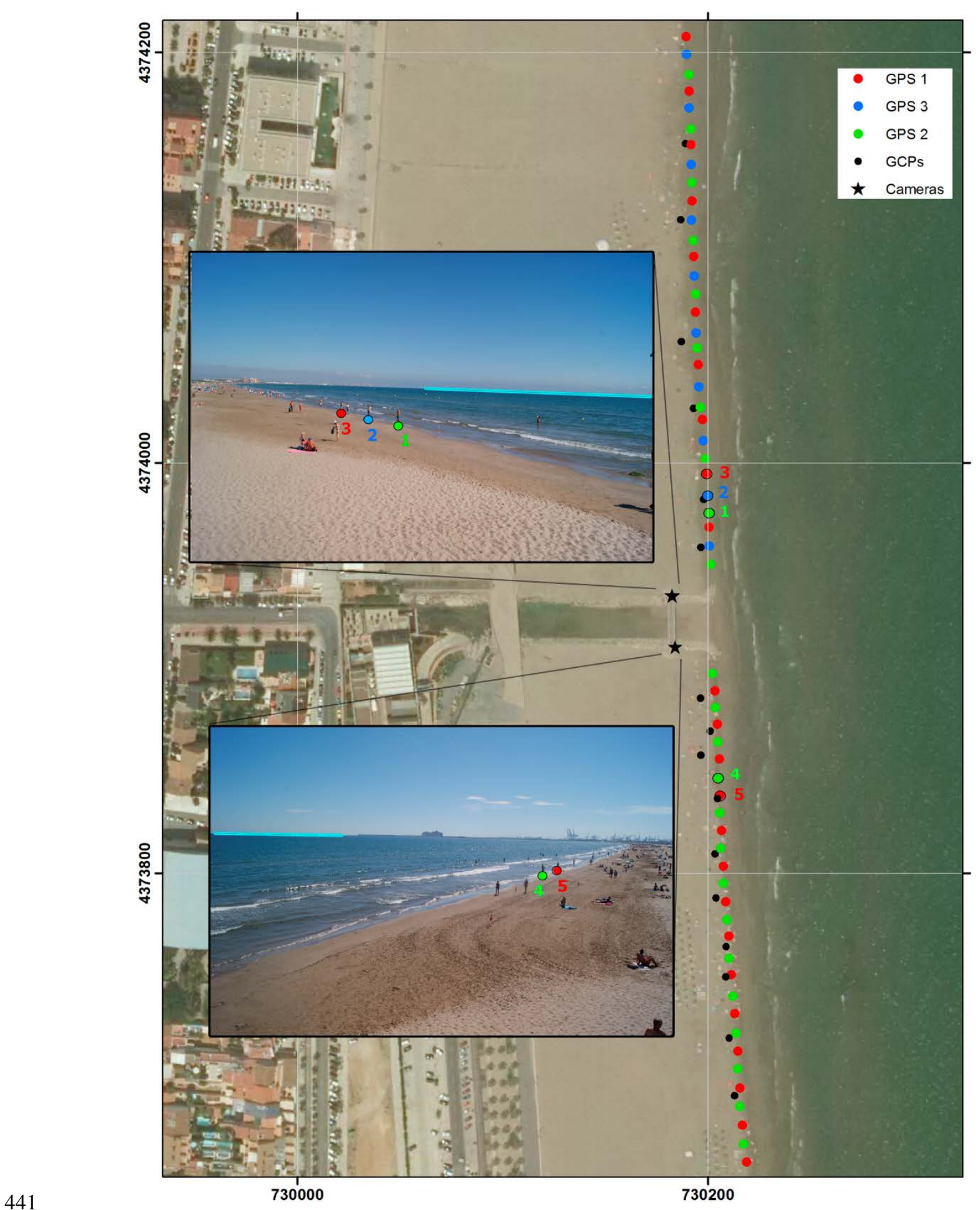

Fig. 3. Mapping GPS data (GCPs and shoreline checkpoints) and two examples of photos acquired for the assessment at Patacona beach. This figure shows the procedure carried out where each shot is performed at the same time as the GPS trackers (three for the north and two for the south) record shoreline positions (checkpoints tagged and numbered) and subsequently evaluated on the rectified photos. The extension of a usable horizon is marked with a blue line. Grid coordinates: GCS_ETRS89 UTM. 


\subsection{Improvement of camera positioning by adding the horizon constraint regardless of whether IOP is} known or not

The aim of this subsection is to establish the improvements and differences achieved by the influence of the horizon constraint in the spatial resection procedure (results of step 2c). For this reason, the resulting performances are achieved by applying C-Pro in various ways, that is, setting and not setting the IOP (depending on whether these are considered as unknown or known) and with and without including the horizon equations in the fitting.

Validating the final EOP for each test at Magoito and Patacona beaches, Table 1 summarizes the differences between these resulting camera coordinates against those accurately measured by GPS. To clarify, the camera positioning error is:

$$
\text { Camera positioning error }(C P E)=\sqrt{\left(X_{C c_{G P S}}-X_{C c}\right)^{2}+\left(Y_{C c_{G P S}}-Y_{C c}\right)^{2}+\left(Z_{C c_{G P S}}-Z_{C c}\right)^{2}}
$$

being $\left\{\mathrm{X}_{C_{C}}, Y_{C_{C}}, \mathrm{Z}_{C_{C}}\right\}$ and $\left\{X_{C_{G P S}}, Y_{C_{C_{G P S}}}, Z_{C c_{G P S}}\right\}$ the camera position coordinates obtained by C-Pro and RTKGPS techniques respectively.

\begin{tabular}{|c|c|c|c|c|}
\hline & & \multicolumn{3}{|c|}{ CPE (m) } \\
\hline & & \multirow{2}{*}{ Without horizon constraint } & \multicolumn{2}{|c|}{ With horizon constraint } \\
\cline { 4 - 5 } & & & Case two points & Case three points \\
\hline \multirow{2}{*}{$\begin{array}{c}\text { Magoito } \\
\text { beach }\end{array}$} & With calibrated IOP & 1.517 & 1.037 & 0.972 \\
\cline { 2 - 5 } & With unknown IOP & 19.780 & 1.848 & 1.827 \\
\hline \multirow{2}{*}{$\begin{array}{c}\text { Patacona } \\
\text { beach }\end{array}$} & With calibrated IOP & 0.847 & 0.566 & - \\
\cline { 2 - 5 } & With unknown IOP & 3.395 & 0.742 & - \\
\hline
\end{tabular}

Table 1. CPE (m) achieved using (11) at Magoito and Patacona beaches. Errors are obtained by comparing the camera position coordinates calculated by C-Pro against those measured by GPS. Results for Patacona beach are the average values in each assessment from all errors shown in Fig. 4.

It is observed that when the camera elevation is higher - as occurs in Magoito with a camera elevation of 35.6 $\mathrm{m}$ in comparison with the elevation of Patacona that ranges from 4.7 or $6.8 \mathrm{~m}$ - the uncertainty of the IOP causes system inconsistency and overstated positioning error. Results of Table 1 reveal the usefulness of the horizon constraint in step 2 of C-Pro. For both cases, with calibrated and unknown IOP, the horizon improves the convergence. However, it is in this last case when it becomes even more necessary to use the horizon to obtain a usable camera position. Regarding the differences between both horizon approximations, the Magoito beach image has a full view of the horizon and the approximation achieved by three points forming a circumference leads to slightly better results. At Patacona beach, the horizon curve is built from only two points because the horizon is just seen in half of the photo and, consequently, its definition using three nearby points would not be rigorous. 


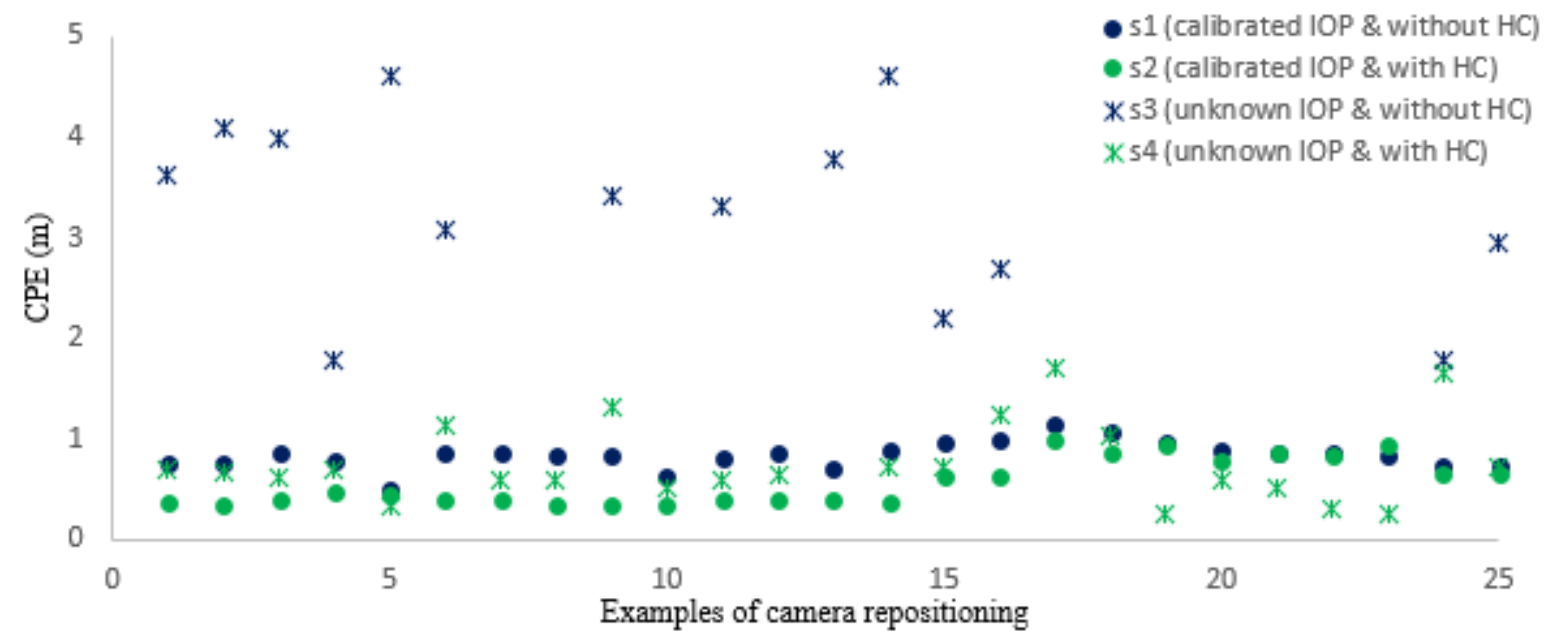

498

Fig. 4. CPE obtained using (11) for 25 different performances and calculated following four different procedures for Patacona beach. Green indicates the use of the horizon constraint in the adjustment, whereas blue represents its absence. Moreover, the asterisks represent those results where the adjustment is made using free IOP and the dots where IOP are obtained by camera calibration.

Fig. 4 shows the results obtained for the 25 performances at Patacona beach where the least squares solution from step 2 of C-Pro has converged. CPE verifies the usefulness of including the horizon constraints (A.26)(A.27) and (3) to obtain an accurate solution for the camera location regardless of whether the IOP are known by calibration or not. It is noteworthy that in most cases when the IOP are unknown, the system solution does not converge unless we introduce the equations of the horizon constraint. In other cases, although the system converges, the achieved solution is not useful and the horizon equations help to obtain it with greater accuracy with errors of within $2 \mathrm{~m}$ in camera position (results of s4 in Fig. 4).

Therefore, thanks to the methodology implemented in this paper, and despite photogrammetry weaknesses when the IOP are unknown, spatial resection of C-Pro achieves accuracies that are in the range of those obtained when all the parameters are under control - an average CPE of $0.742 \mathrm{~m}$ versus $0.566 \mathrm{~m}$ respectively. However, the magnitude of this error is going to be strongly related with other conditioning factors. The main factors are: the GCP distribution; the degree of success produced during the detection in the photo of GCPs and horizon points; and the difficulties in image geometry. 

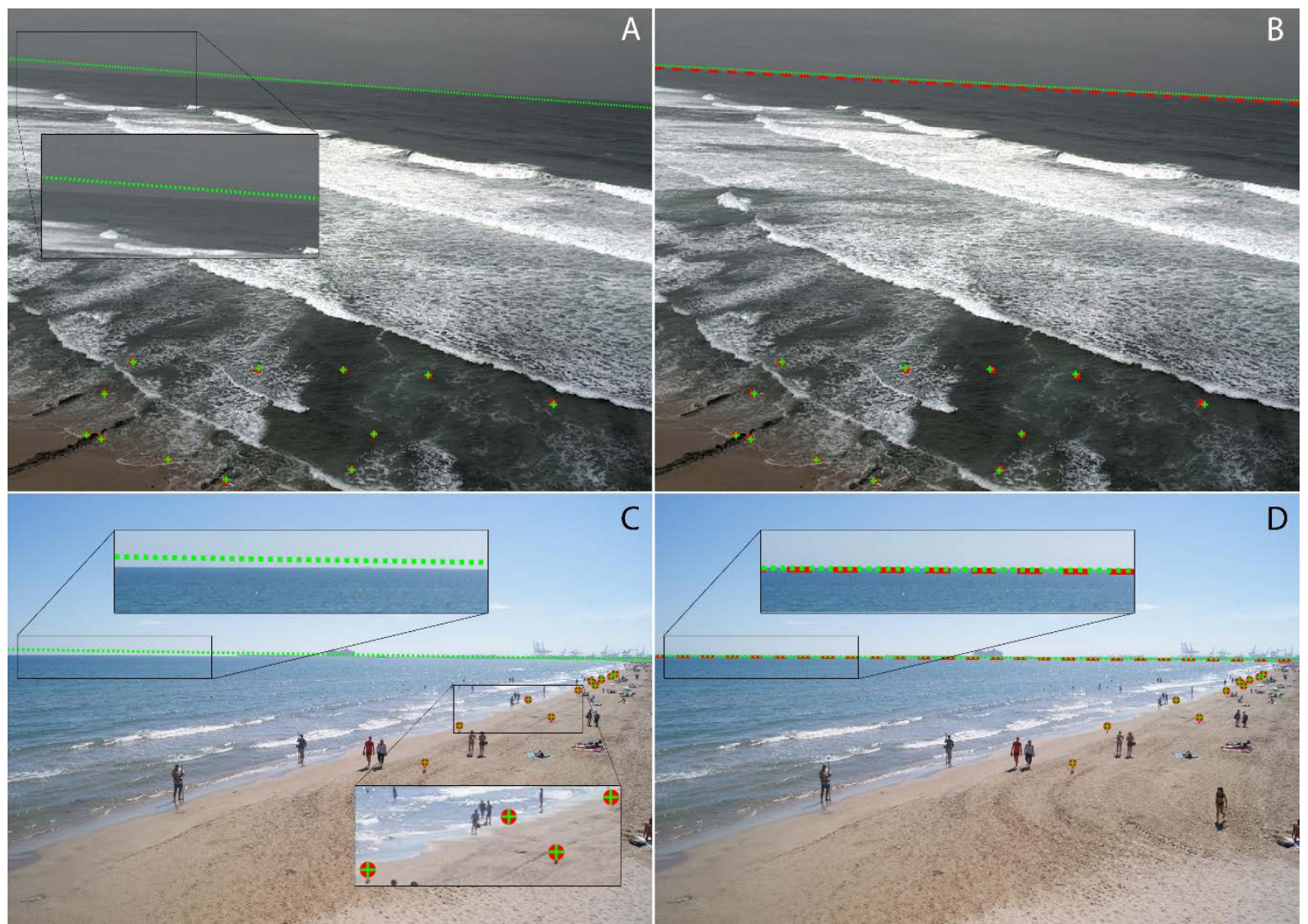

Fig. 5. Examples of the system convergence for two performances carried out at Magoito (A and B) and Patacona (C and D) beaches. The observed GCP positions and the observed horizon are shown in red respectively by points and a dashed line - adjustment input data - whereas the calculated position of these features (system solution after the iterative process) is shown in green by crosses for GCP and a dotted line for the horizon. A) and C) Wrong resulting convergence achieved without including the horizon constraint in the adjustment (the observed horizon is not involved); B) and D) Best convergence reached using the horizon as a system constraint (both with unknown IOP).

It is difficult to compute the error made in calculating the angular parameters $\{\omega, \varphi, \kappa\}$. Nevertheless, the quality of these can be analyzed through the location of the control points and the horizon line after applying step 2d) of C-Pro, as Fig. 5 shows at Magoito and Patacona beaches. Carrying out the spatial resection with unknown IOP, Figs. 5A and 5C show that in the adjustment made without including the horizon constraint, the resulting camera orientation is mistaken despite the convergence (the green line is wrongly indicating the calculated horizon direction). However, the resulting camera angles are more accurate when the horizon constraint is included in the adjustment that defines a correct horizon line. Thus, in this last case, both observed and calculated horizon lines - shown respectively in red and green in Figs. 5B and 5D - indicate an equivalent direction of the horizon.

The next assessment is made to discover in more detail the way in which the system is converging into a specific solution in accordance with to the weight assigned in the two horizon equations of (3). The system is then solved several times for the same example - using the same photo and its associated GCP file - and adding weight each time to the horizon equations. The usefulness of the horizon constraint will be studied in Fig. 6 by analyzing the CPE of seven photos from Patacona beach. These seven cases show, even when horizon equations are not intervening (cases with a weight equal to zero), that the system always converges in a solution for the spatial resection even when the IOP are unknown. However, the achieved CPE decreases as 
the horizon equations gain more weight (see Fig. 6). This is evidenced in those cases where the adjustment has been carried out with nine free parameters (examples with unknown IOP) and because the horizon constraint has not considered that the CPE are higher than $2 \mathrm{~m}$. Additionally, in these cases it is interesting to note that the minor CPE occurs for horizon weights equal to $10^{2}$ and $10^{4}$. This improvement in the accuracy of the camera coordinates happens while the solution for the rest of the parameters (three camera angles and IOP) worsens. The system is less stable when the IOP are unknown and cannot find a valid solution for the entire set of parameters until the weight of the horizon increases.

The CPE remains unchangeable from a weight equal to $10^{12}$ for both horizon equations although it is almost stabilized from using a weight equal to $10^{8}$. Thus, horizon equations will be added in the adjustment in step 2c) of C-Pro with a designated weight equal to $10^{12}$ and can be modified by the user.

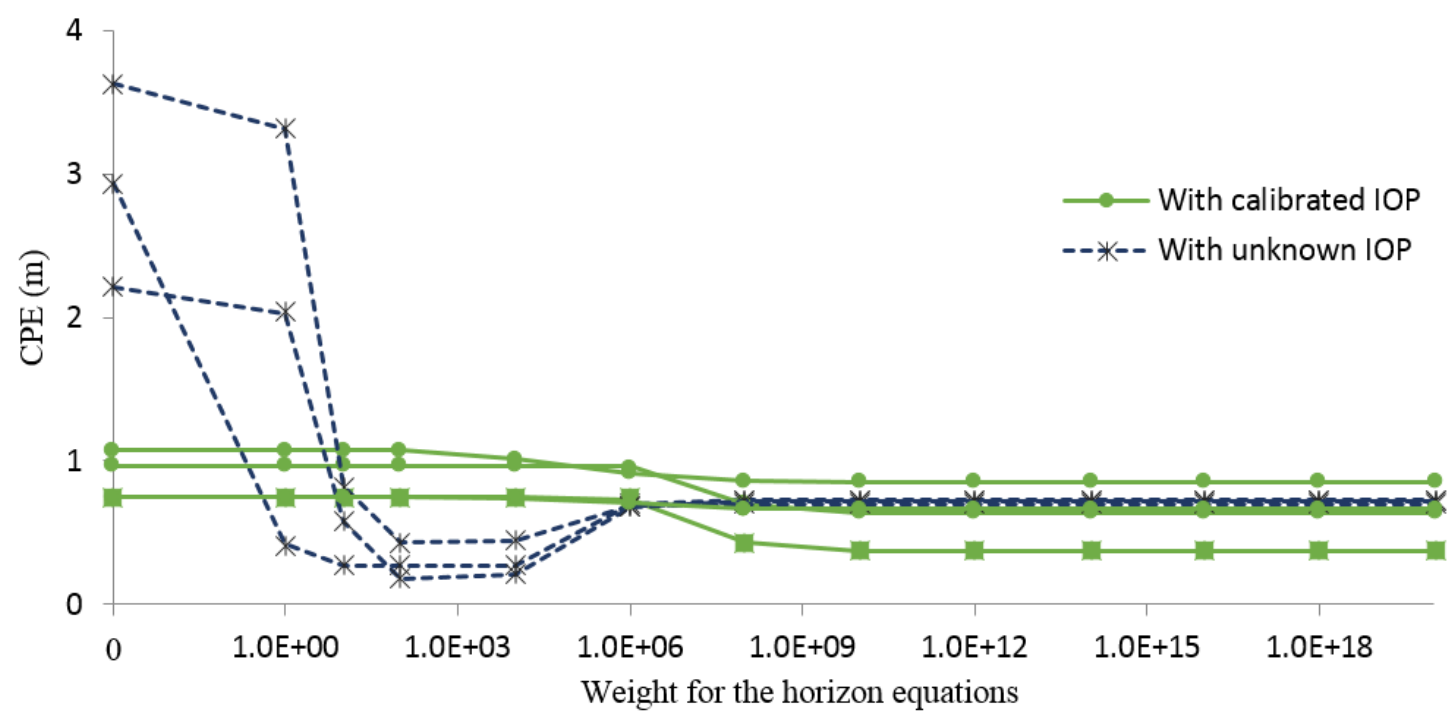

Fig. 6. CPE (m) obtained with C-Pro in seven different photos from Patacona beach are consistent with the assigned weight value in the two horizon equations of (3). The elevation of the camera ranges between $4.69 \mathrm{~m}$ and $6.81 \mathrm{~m}$.

\subsection{Usefulness and differences between both horizon approximations}

After checking the improvement achieved by introducing the horizon constraint in the spatial resection process, it is important to establish the quality of the two horizon approaches developed in this paper. Therefore, this section analyzes their potential and limitations when these geometric equations are constraining the fitting. With regards to the definition of the horizon equations, it is expected that the correct choice of one or another approach (with two or three points) was conditioned by the percentage of horizon that appears in the photo.

The acquisition of a large set of photos for two days at El Saler beach with different percentages of horizon contained on the photographs enables us to analyze the error achieved in the spatial resection solution depending on the horizon approximation used during the process. Assessments are made by following the methodological protocol considering the IOP as unknown. Summarizing the results (see Table 2), we observe unacceptable results regardless of the camera location, and regardless of whether the camera is pointing north (CNi) or south (CSi), when the horizon constraint is not considered because the fitting does not converge (or it converges on a wrong solution). 


\begin{tabular}{|c|c|c|c|c|c|}
\hline & & \multirow{2}{*}{$\begin{array}{l}\text { Without horizon } \\
\text { constraint }\end{array}$} & \multicolumn{2}{|c|}{$\begin{array}{c}\text { With horizon } \\
\text { constraint }\end{array}$} & \multirow{2}{*}{$\begin{array}{l}\text { \% horizon seen } \\
\text { in the photo }\end{array}$} \\
\hline & & & $\begin{array}{l}\text { two } \\
\text { points }\end{array}$ & $\begin{array}{l}\text { three } \\
\text { points }\end{array}$ & \\
\hline \multirow[t]{7}{*}{$\begin{array}{l}25 \text { May } \\
2016\end{array}$} & CN1 & Without convergence & 0.139 & 0.930 & 51.5 \\
\hline & CN2 & 15.029 & 1.478 & 1.359 & 100 \\
\hline & CN3 & Without convergence & 0.956 & 0.826 & 100 \\
\hline & $\mathrm{CN} 4$ & 6.854 & 0.635 & 0.393 & 90 \\
\hline & CS1 & 15.118 & 0.568 & 1.105 & 61.7 \\
\hline & CS2 & 3.839 & 0.976 & 0.896 & 100 \\
\hline & CS3 & 2.769 & 2.041 & 1.891 & 89 \\
\hline \multirow{7}{*}{$\begin{array}{l}17 \text { June } \\
2016\end{array}$} & CN1 & Without convergence & 0.114 & 1.460 & 51.5 \\
\hline & $\mathrm{CN} 2$ & 17.516 & 2.164 & 2.010 & 100 \\
\hline & CN3 & Without convergence & 1.092 & 0.872 & 100 \\
\hline & $\mathrm{CN} 4$ & 6.332 & 0.586 & 0.491 & 90 \\
\hline & CS1 & 12.989 & 1.472 & 2.198 & 61.7 \\
\hline & CS2 & 1.649 & 0.729 & 0.669 & 100 \\
\hline & CS3 & 12.05 & 1.616 & 1.077 & 89 \\
\hline Average & & 9.414 & 1.227 & 1.048 & \\
\hline
\end{tabular}

Table 2. CPE (m) obtained without known IOP in various cases, with or without the horizon constraint at El Saler beach. Horizon is approximated using two or three image points, respectively. Last column shows the percentage of the horizon visible in the image and not hidden behind other elements. CNi and CSi indicate respectively north and south - depending on where the camera points.

The errors in the camera repositioning for both days indicate that the horizon approach obtained by three points leads to better results unless the horizon covers less than $62 \%$ of the photo area - as occurred in both CN1 and CS1 photos where the horizon remain partially hidden by the Port of Valencia (see example in Fig. 2). Predictably, the horizon approach calculated by three points is more realistic, but is also more sensitive and requires a significant distance between points to define a descriptive horizon circumference. In general, when the horizon just appears in a proportion less than $75 \%$ of the photo, the approximation must be defined by two points.

Representing the results graphically in Fig. 7, the pattern followed by the CPE repeats for both studied days. The most rigorous solutions for the spatial resection process are achieved by using the horizon approximation with three points, with smaller errors in the camera positioning until the percentage of the apparent horizon in the photo exceeds a specific limit as occurs in CN1 and CS1 (small circles). It is in these cases when the pattern of error reverses and the errors caused by using the approximation with two points becomes smaller.

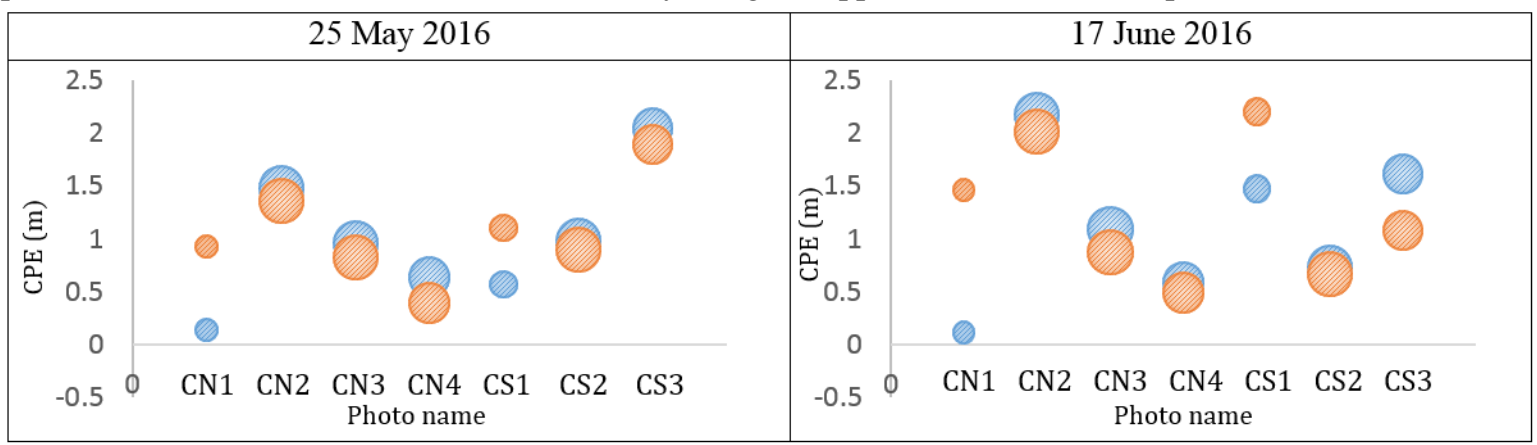

Fig. 7. CPE (m) obtained by using the two horizon approximations in seven photographs from El Saler beach 
and for two different days. Blue represents the results achieved by using the two point horizon approximation (case a), and red indicates those obtained by the horizon approximation defined with three points (case b). The circle size increases with the proportion of horizon visible in the photo.

Moreover, by calculating the differences between the CPE obtained by using both horizon approximations we realize that the negative values correspond with those cases where the horizon is scarce in the photo. A clear relation exists when comparing these differences against the percentage of horizon, reaching $R^{2}=79.72 \%$ in the linear fit (Fig. 8) and $R^{2}=90.25 \%$ by fitting a second order polynomial model.

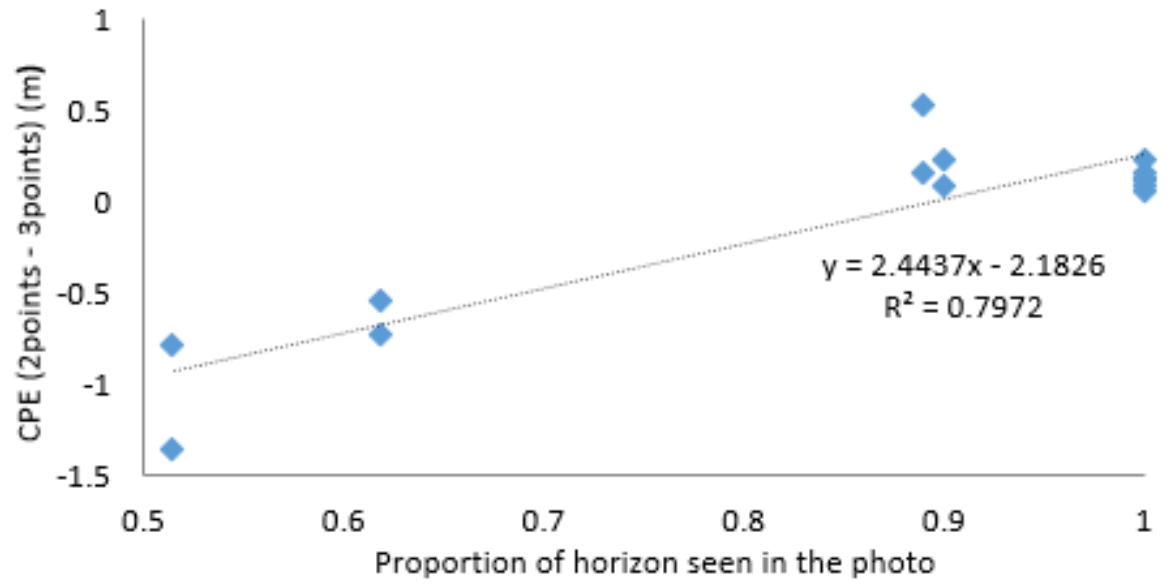

Fig. 8. CPE of Fig. 7 as a function of the proportion of horizon seen in the photo.

With regards to the conclusions obtained through these experiments, it is easy to know why at Magoito beach (where the photos contain a full view of the horizon) the approximation achieved by three points led to slightly better results as seen in Table 1. Two-point horizon approximation was used at Patacona beach because the horizon appeared in less than half of the image.

\subsection{Analysis of errors after the image rectification process}

The final accuracy of the system depends on many factors. This subsection is focused on managing the errors related to the image rectification process through the implementation of a rigorous algorithm (after step 3). To assess the overall positional accuracy for the rectified images in the three study areas, several terrain points those not used to solve the geometry and termed checkpoints - were computed solving (10) and comparing their projected computed coordinates $(X, Y)$ against GPS coordinates after step 3 of C-Pro. Formula (12) calculates this error (which is composed by both cross-shore and longshore components).

$$
\text { Positional error }=\sqrt{\left(X_{G P S}-X\right)^{2}+\left(Y_{G P S}-Y\right)^{2}}
$$

Moreover, regarding the camera position and the focal length, the pixel footprint will be calculated to obtain the dimension of each pixel in the terrain space.

The first analysis was made at Magoito beach using the orientation parameters achieved in the best case with calibrated IOP and the horizon constraint approximated by three points (see Table 1). Despite the $0.97 \mathrm{~m}$ of error recorded in the camera repositioning, the checkpoints - 65 to $98 \mathrm{~m}$ distant from the camera - are positioned with an average accuracy of $0.201 \mathrm{~m}$. Moreover, Fig. 9 shows a slight relation between each positional error and the distance to the camera because of the perpendicular GCPs distribution relative to the camera position (remember Fig. 1 in the data section). 


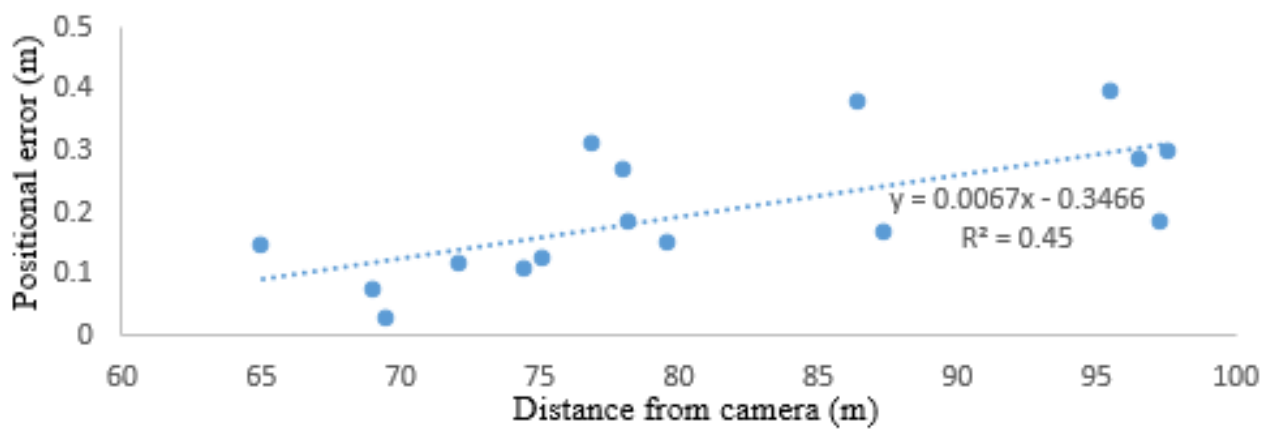

614

615

616

617

618

619

620

621

622

623

624

625

626

627

628

629

630

631

632

633

634

635

636

637

638

639

640

641

642

643
Fig. 9. Scatter plot that relates the behavior of the positional errors in the checkpoints at Magoito beach relative to their distance from the camera.

The error is dominated by the cross-shore component through the target area and, as expected, is closely related with a pixel footprint smaller than $0.5 \mathrm{~m}$.

The second experiment at El Saler beach (see Table 2) also includes a study of projected errors in the checkpoints using the horizon approximation in the spatial resection system with unknown IOP. It is important to remember that the photos will be different every day because we are not working with a fixed camera. As a result, the GCPs image coordinates, the resulting spatial resection, and the final image rectification, will change.

Fig. 10 shows that differences between averages of positional errors using each of the two horizon approximations are smaller than $0.1 \mathrm{~m}$. Again, the results indicate that in most cases, those errors are lower when the horizon constraint is obtained following the approximation with three points. If the horizon visibility requirements are overtaken, we should then follow the methodology where the horizon curve is approximated as the tangent line to a circumference. Furthermore, Fig. 10 proves an increasing trend of the positional errors with respect to the camera distance. However, up to a distance of $200 \mathrm{~m}$, errors in the checkpoints respond to a similar behavior for both horizon approximations. Considering only the points with distances to the camera of less than $200 \mathrm{~m}$, the average positional error for the 25 May is equal to $0.286 \mathrm{~m}$ using the horizon approximation with two points and $0.283 \mathrm{~m}$ using the three-point approximation. For the 17 June, both results are equal to $0.275 \mathrm{~m}$ and $0.273 \mathrm{~m}$, respectively. However, from $200 \mathrm{~m}$ to $610 \mathrm{~m}$, the differences in the positional errors obtained for each horizon approach become more apparent. The averaged results verify the success of the horizon approximation with three points with values equal to $0.793 \mathrm{~m}$ and $1.140 \mathrm{~m}$, respectively for 25 May and 17 June, meanwhile using only two points, those values reach, in the same checkpoints, $1.170 \mathrm{~m}$ and $1.670 \mathrm{~m}$ respectively. 


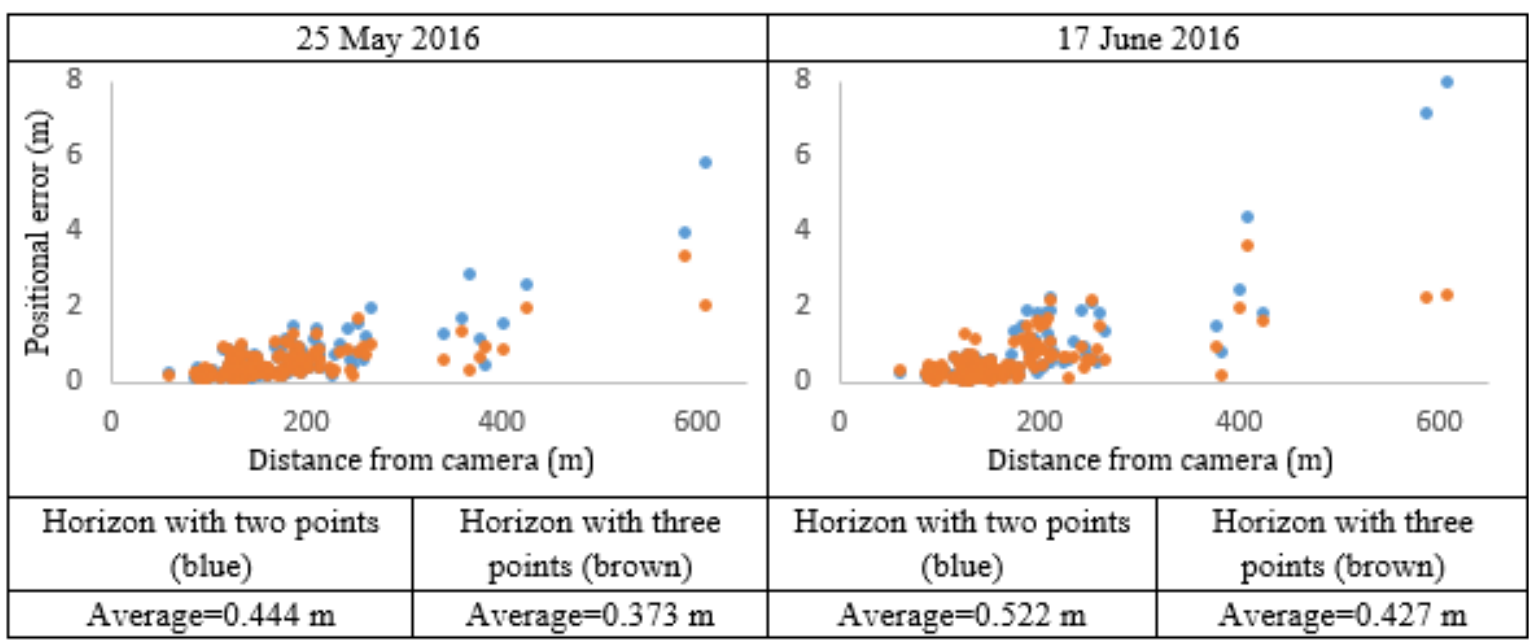

Fig. 10. Variation of the positional accuracy (m) with distance from the camera at El Saler beach depending on the horizon approximations (two or three points) used for spatial resection. The rectification process has been made for the seven different photos in each of the two days described in Table 2 considering unknown IOP.

Furthermore, it is important to establish the differences in magnitude between both cross-shore and longshore error components (see Fig. 11) depending on the phenomenon analyzed. For instance, when the photos are used to extract longitudinal coastal features such as the water/land border, the positional accuracy will be dominated by the cross-shore component. Comparing a surveyed RTK-GPS shoreline and another obtained from the rectified images as illustrated in Fig. 12 - projected on the corresponding sea level elevation - the root mean square error (RMSE) was $1.482 \mathrm{~m}$ and $1.645 \mathrm{~m}$ for 25 May and 17 June respectively. These encouraging results are in line with other similar works such as Taborda and Silva (2012) where the swash line position is rectified with an RMSE of $1.4 \mathrm{~m}$, and Bracs et al. (2015) where the surfcam-derived shorelines are calibrated against Argus shorelines with an error less than $1.9 \mathrm{~m}$.

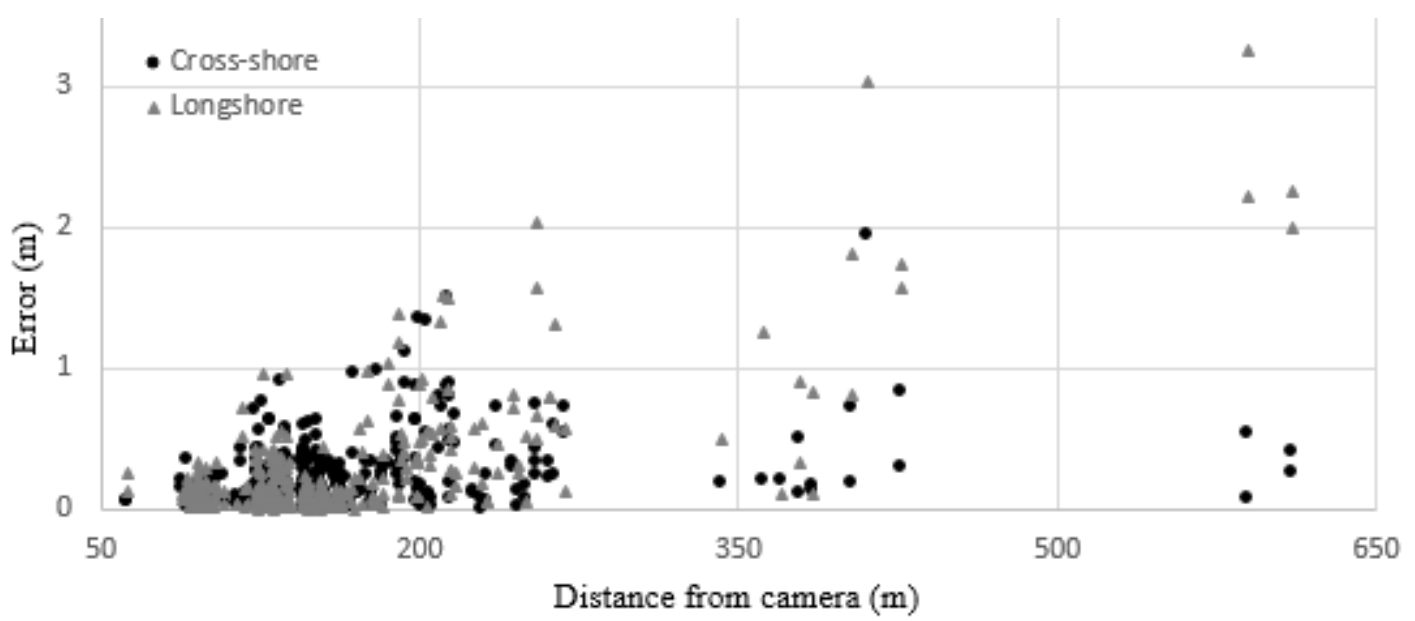

Fig. 11. Cross-shore and longshore errors for data used in Fig. 10 at El Saler beach. The errors are obtained when carrying out the best spatial resection solution obtained in each case. 


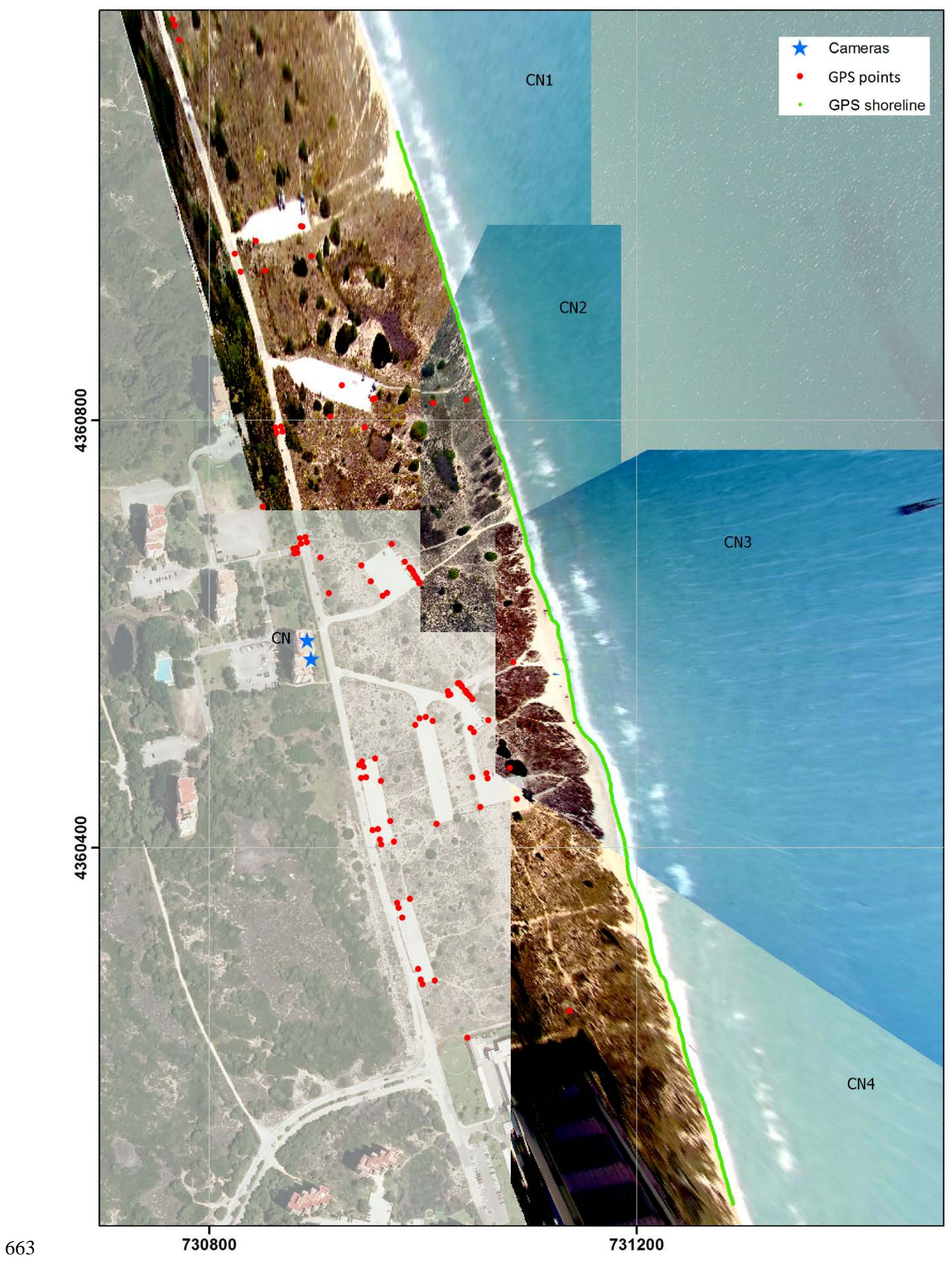


664

665

666

667

668

669

670

671

672

673

674

675

676

677

678

679

680

681

682

683

684

685

686

687

688

689

690

691

692

693

694

695

696

697

698

699

Fig. 12. Projection map with the rectified photos of 25 May 2016 for El Saler beach shown over an orthophoto taken from 2010 PNOA sources. The four different shots are obtained from the CN position (north camera). It is important to know that the projection is made at $0.129 \mathrm{~m}$ above mean sea level - as near in time with the photos as possible - whereas the RTK-GPS shoreline (green line) has an average elevation of $0.11 \mathrm{~m}$. Grid coordinates: GCS_ETRS89 UTM.

For the last analysis, at Patacona beach, the evaluation of the image rectification process was made by checking the coordinates of several measured terrain points to thoroughly analyze the longitudinal component of error (longshore positional accuracy). The acquisition process measuring points by progressively moving the GPS trackers towards the shoreline while each photo was taken is explained in the data section. Furthermore, this data is used to continue with the analysis of errors depending on the spatial resection adjustment carried out (cases s1 to s4) and which is expected to be in line with the CPE previously shown in Table 1.

When the adjustment was computed by just six free parameters (data pointed in Fig. 11), the average error was $2.48 \mathrm{~m}$ or $4.29 \mathrm{~m}$ depending on whether the horizon constraint was considered (s2) or not (s1). The horizon approximation has been computed with only two points as in Fig. 13. Moreover, when the IOP are unknown (data dashed in Fig. 11), the errors were worse and reached $3.91 \mathrm{~m}$ with horizon constraint and 5.69 $\mathrm{m}$ without. These higher magnitudes of error, compared with those obtained in the above two beaches, are consequences of the low-elevation cameras (only $4.7 \mathrm{~m}$ to $6.8 \mathrm{~m}$ high) and the pixel rectification error associated with obliquity. This fact greatly complicates the detection of the checkpoints in the photography with clarity being rapidly lost with distance. These difficulties are also linked with the limitations of the camera optics. An initial study analyzing both cross-shore and longshore errors (see Fig. 13) indicates that some problems in the manual detection of the checkpoints are expected - with errors in their real positions because of visual obstructions such as beach berms. This fact is very influential in longshore error as it is more sensitive to the distance from the camera and where a single pixel at $200 \mathrm{~m}$ distance means $1 \mathrm{~m}$ of error in the geographic space. However, the valid magnitudes for the cross-shore components indicate the ability and solvency of the methodology for detecting coastal indicators.

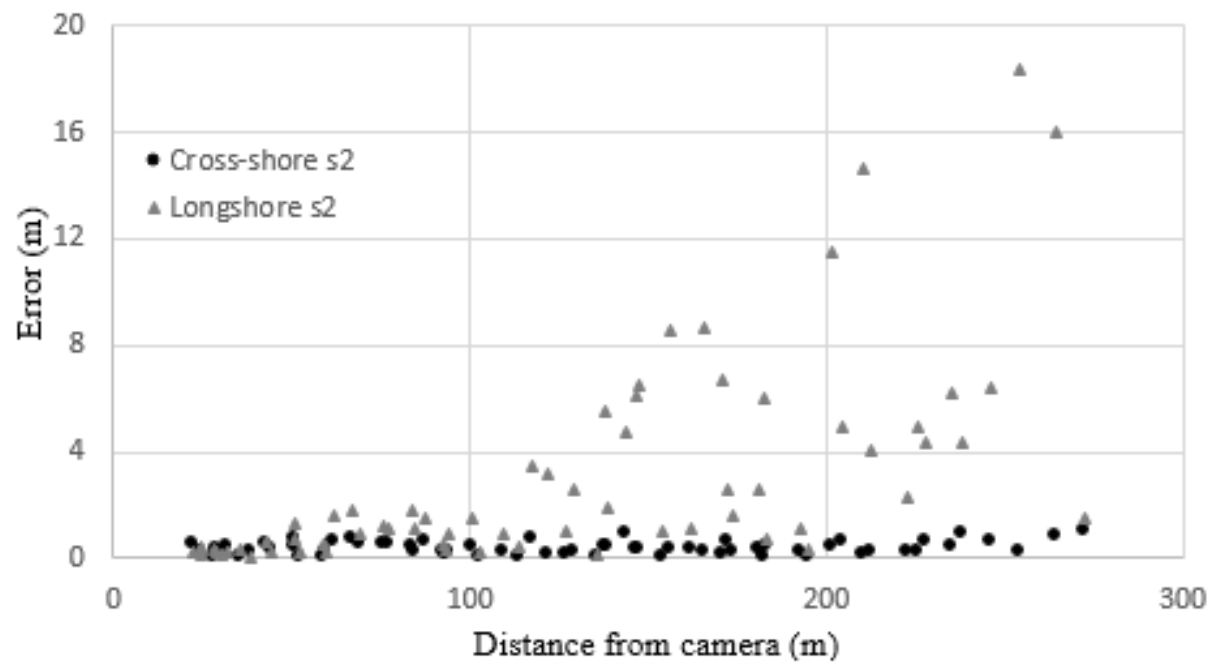

Fig. 13. Cross-shore and longshore positional accuracies after the rectification procedure (calibrated IOP \& with HC) obtained for some GPS points measured on the shoreline at Patacona beach.

The next analysis focuses just on those points located less than $200 \mathrm{~m}$ from the camera, discarding the others as reliable for computing the overall error. By averaging these selected points, we obtain positional accuracies reaching $1.68 \mathrm{~m}$ when the image rectification is computed following s2, $2.86 \mathrm{~m}$ with s1, $2.91 \mathrm{~m}$ with s4, and $4.56 \mathrm{~m}$ in the worst case through $\mathrm{s} 3$. 


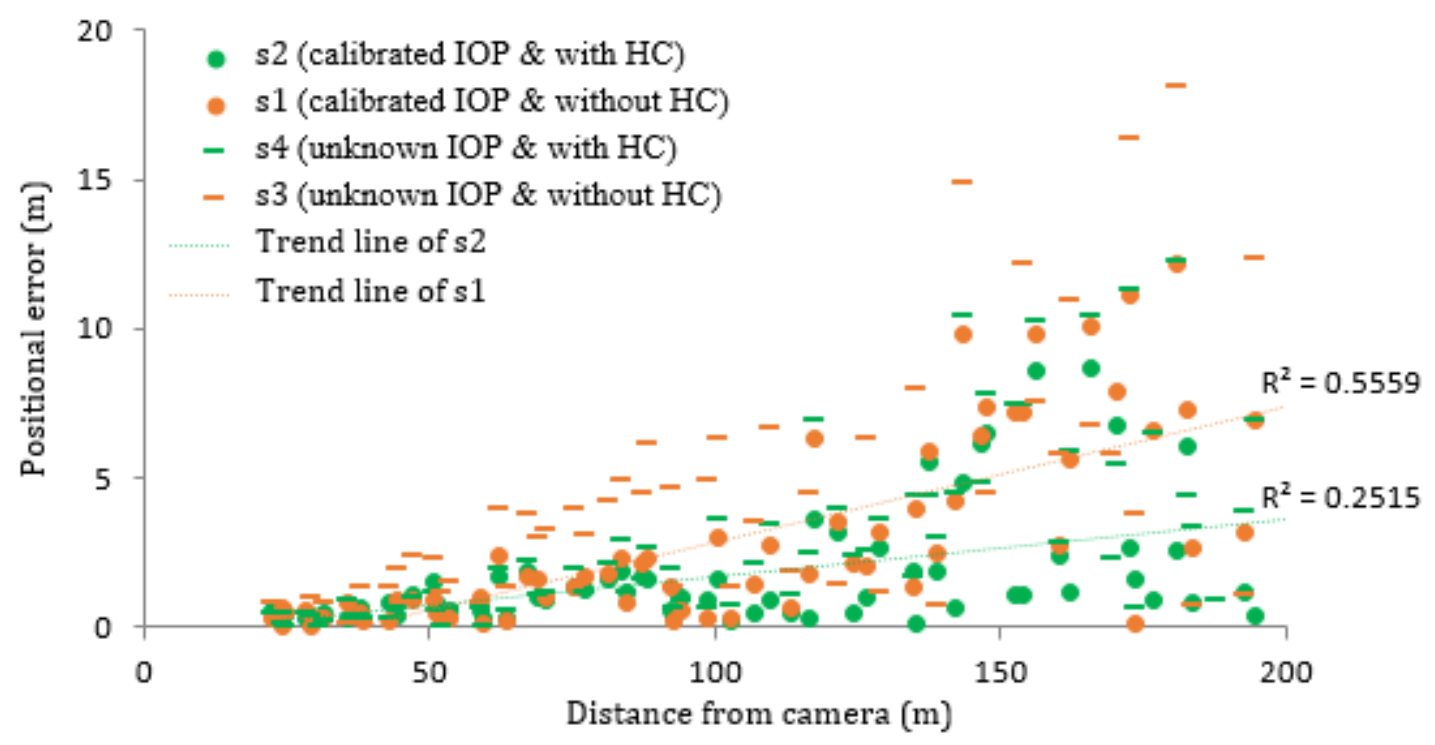

Fig. 14. Variation of the positional error with distance from the camera depending on the methodological process carried out. We detected points in 25 photos at Patacona beach (spatial resection was already analyzed in Fig. 4). Remember that the photos were taken with low-elevation cameras that range from $4.7 \mathrm{~m}$ to $6.8 \mathrm{~m}$ high.

Results verify that despite not knowing the IOP, similar averaged errors obtained for s1 and s4 mean that the use of the horizon constraint provides solutions as valid as those obtained when the initial parameters are calibrated. Moreover, we realize in Fig. 14 that positional errors have a strong dependence on camera distance. Longitudinal errors grow excessively when the horizon constraint is not included $\left(R^{2}=55.59 \%\right)$ whereas its use slows this fact $\left(\mathrm{R}^{2}=25.15 \%\right)$ by leading the rectified image to a proper alignment.

\section{Discussion and conclusions}

This paper has described a new coastal projector monitoring system called C-Pro which uses terrestrial photogrammetry to project a photograph in a georeferenced plane. The main novelty, compared with other methods previously described in the literature, lies in the definition of a mathematical formulation that incorporates information provided by the location of the horizon curve in the image. For this, the roll and pitch rotation angles have been computed from an approximation of the horizon curve to define the transformation from image vectors to terrain vectors. This describes the change between coordinate reference systems: from object space to image space. The relationship between these rotation angles and the EOP of the camera leads to the horizon equations. Thus, an initial solution of these angular parameters can be obtained from horizon equations, which are also used in the repositioning process of the camera as constraints providing two degrees of freedom. Oreifej et al., (2011) already exploited the horizon line in terms of providing a unique unambiguous solution for recovering the UAV camera motion.

C-Pro has been applied to three different coastal areas using two cameras located at several elevations ranging from $4.7 \mathrm{~m}$ (Patacona beach) to $43 \mathrm{~m}$ (El Saler beach). Results have shown the improvement that occurs in estimating the camera positioning when adding the horizon constraints, especially in the case of using cameras with unknown IOP. Sometimes, the iterative least squares fitting - over the linearized collinearity equations - does not converge unless the horizon constraints are used. In other cases, although the system converges, the achieved solution is not useful and the horizon equations help reduce errors in camera positioning and angular parameters $\{\omega, \varphi, \kappa\}$. The quality of the spatial resection has also been analyzed by coloring the resulting image location of the control points and the horizon line using the calculated parameters after each iteration. Errors are smaller when weights assigned to horizon constraints in that refinement process are much higher than those given in equations associated with GCPs. 
Image coordinates of three points are used to approximate the horizon curve. However, results have shown that a horizon approach with two points is more accurate in situations in which only a part of the horizon is seen in the image. The horizon approach calculated by three points is more realistic but is more sensitive and requires a significant distance between points to define a descriptive horizon circumference.

Some terrain points with known GPS coordinates, which have not been involved in the setting as GCPs, were also computed with C-Pro to assess the overall positional accuracy over the rectified images in the three coastal areas. At Magoito beach, the checkpoints situated less than $65 \mathrm{~m}$ from the camera were positioned with an averaged accuracy of $0.2007 \mathrm{~m}$. Moreover, errors were less than $0.5 \mathrm{~m}$ for points located within 100 $\mathrm{m}$ of the camera. Those errors of projection were obtained after placing the camera with an elevation of 35.6 m, using C-Pro with calibrated IOP, and approximating the horizon constraint with three points.

Errors at checkpoints increase slightly when the IOP are unknown as is assessed in El Saler beach where the camera was located at the top of a building $43 \mathrm{~m}$ high. However, acceptable results are obtained by considering the horizon approximation in the spatial resection system, computed following the approximation with three points. Those results have been obtained with a non-fixed camera taking 14 photos in two days. Consequently, as the GCPs image coordinates will change, the resulting spatial resection will also differ for every photo. Considering the checkpoints whose distances to the camera were less than $200 \mathrm{~m}$, the average error was equal to $0.283 \mathrm{~m}$ on the first day and $0.273 \mathrm{~m}$ on the second day.

At Patacona beach, the camera is located at an elevation ranging between 4.7 and $6.8 \mathrm{~m}$. In this case, we analyze the longshore error through an experiment measuring shoreline points until a distance of less than 200 $\mathrm{m}$ from the camera is found that is considered as reliable for computing that error. Positional accuracies reached an average positional error equal to $1.68 \mathrm{~m}$ when the image rectification was computed using calibrated IOP and $2.91 \mathrm{~m}$ when considering unknown IOP. The horizon approximation with two points was used in these cases because the horizon appeared in less than half of the image. Thus, the use of the horizon constraint has enabled us to obtain valid solutions even in cases when it is not possible to obtain the IOP by camera calibration (or other complicated photogrammetric conditions such as low camera elevations were present).

The methodology developed in this work enables accurately projecting a coastal photograph - or any element detected in it - on a georeferenced plane, even if the photo was taken by a camera with unknown IOP and located at a less than $7 \mathrm{~m}$ high. Encouraging results (similar to those obtained by Taborda and Silva, 2012) which are able to define the shoreline with an RMSE of less than $1.5 \mathrm{~m}$. Its implementation in C-Pro, through formulas detailed in this paper, makes it a robust and low-cost tool that can work with any photograph taken by a conventional camera of a coastal segment with the horizon included. In this paper, the horizon points have been marked manually but future works applying C-Pro will use techniques that automatically track the horizon (Bracs et al., 2015; Oreifej et al., 2011).

The application of C-Pro will produce valuable scientific information from numerous cameras and video cameras located along coastlines worldwide. Although in principle these recreational cameras were set for other non-metric goals, they can now also be useful for measuring beach indicators for better planning and managing coastal resources.

\section{Acknowledgements}

This study is part of the $\mathrm{PhD}$ dissertation of the first author, which is supported by a grant from the Spanish Ministry of Education, Culture and Sports (I+D+i 2013-2016). Two other projects participated: one from the Spanish Ministry of Economy and Competitiveness (CGL2015-69906-R) and the other from the Valencia Regional Government (AICO/2015/098). The Department of Geology, Faculty of Science of the University of Lisbon provided Magoito data thanks to a $\mathrm{PhD}$ stay of the first author under the supervision of Dr. Rui Taborda. The authors gratefully acknowledge the constructive comments provided by Dr. Jaime Almonacid.

\section{Appendix A: Horizon constraint}


The methodology of terrestrial photogrammetry described in this paper is designed for use in coastal imaging systems where the horizon is an element of the photo (meaning the separation between sea and sky). Appendix A describes some mathematical tools that take advantage of the information provided by the horizon line and are useful for the new coastal projector monitoring system (C-Pro), explained in Section 2.

\section{A.1. Image orientation using the horizon}

The calculation of the image orientation with respect to the object space is made through three rotations and three corresponding angles that transform image data into real-world coordinates: $\omega \in[-\pi, \pi], \varphi \in$ $[-\pi / 2, \pi / 2], \kappa \in[-\pi, \pi] . R_{\kappa \varphi \omega}$ consists of the product between an initial rotation matrix in the $\mathrm{Z}$ axis, later in $\mathrm{Y}$ axis, and finally in the $\mathrm{X}$ axis. Thus, $(\mathrm{X}, \mathrm{Y}, \mathrm{Z})$ coordinates of a point in the object reference system focused on the main point of the image can be calculated by knowing its associated coordinates $(x, y, z)$ in the image reference system.

In the following paragraphs the $(\mathrm{x}, \mathrm{y}, \mathrm{z})$ coordinates in the image space are referred to as the principal point $o=\left(x_{0}, y_{0}\right)$ and to simplify formulas in this section, its coordinates are assumed as zero $o=(0,0)$. Both the object coordinates and the image coordinates - originally defined from the upper left corner of the image are translated by establishing its origin in the principal point. However, there are other rotational angles relating both image and object coordinates such as described by Dai et al., 2011 and Rodríguez et al., 2008. We are going to use several of these ideas to define the equations of change between reference systems through new angles.

The definition of these angles is based on the approximation of the Earth's curved horizon as a straight line in the image plane. Two alternatives for the determination of that straight line are proposed in this paper by marking two vanishing points in the image that are as distant as possible; $\mathrm{A}=\left(\mathrm{x}_{\mathrm{a}}, \mathrm{y}_{\mathrm{a}}\right), \mathrm{B}=\left(\mathrm{x}_{\mathrm{b}}, \mathrm{y}_{\mathrm{b}}\right)$, and fulfil that $\mathrm{x}_{\mathrm{a}}<\mathrm{x}_{\mathrm{b}}$. In this paper, the horizon points have been marked manually to ensure that they do not become an added source of error in the methodological assessment.
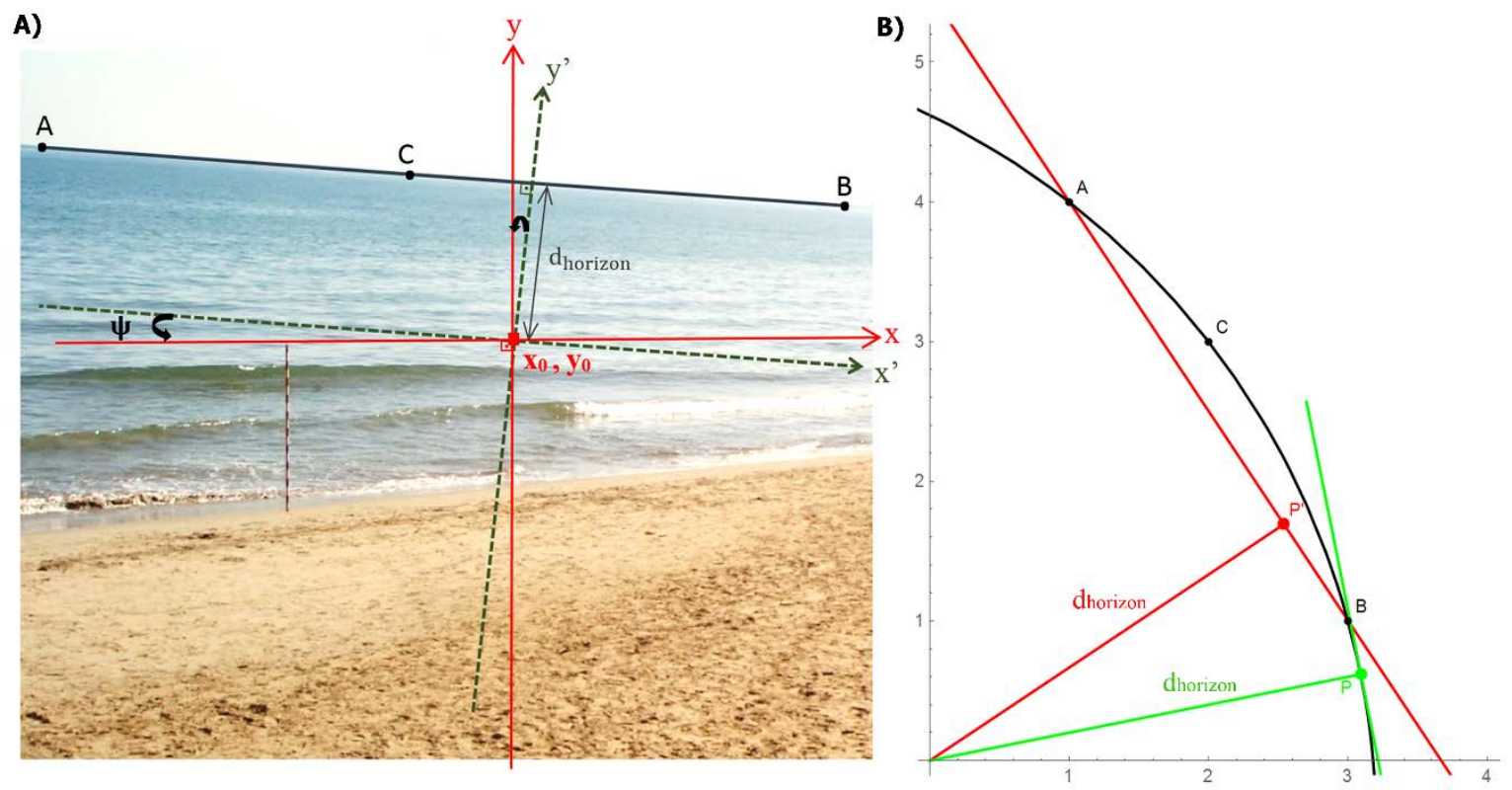

Fig. A.1. Image orientation: A) changing the coordinate system in the image space where the angle $\psi$ is defined; and B) representation of both horizon approximations: shown as a red line following case a) and in green for case b). 
Case a) (two points) The horizon curve is approximated through the line joining both A and B points (colored red in Fig. A.1B).

Case b) (three points) If $A$ and $B$ points are far enough apart, it is possible to find a third point $C=\left(x_{c}, y_{c}\right)$. These three points define a circumference and we compute $\mathrm{P}$ as the point where the minimum distance is reached between the circumference and the principal point of the image plane. The tangent line to the circumference at such point $P$ then approximates the real curved horizon (shown in green in Fig. A.1B).

Note that the horizon approximation with three points is the most realistic. However, it is very sensitive and strongly dependent on the horizon extension seen in the image. If the three points responsible for forming the circumference are too close to each other due to the reduced horizon extension, it will not be reliable and we should proceed using the methodology with two points described in case a). In the results section, the influence of this horizon requirement is analyzed.

Therefore, considering some of the above procedures, the horizon curve is finally approximated through a straight line. Assuring the consistency of a semi-automatic process, we need to have control over the correct order in the manual input of the image coordinates verifying that $\mathrm{x}_{\mathrm{a}}<\mathrm{x}_{\mathrm{c}}<\mathrm{x}_{\mathrm{b}}$ (see Fig. A.1A). Moreover, these should verify that in the $A B C$ triangle, the $C$ internal angle ranges $] 0, \pi[$.

To define the new rotation angles from the calculated horizon line -widely known as roll and pitch angles (Oreifej et al., 2011) - it must be remembered that such a horizon line is parallel to both the object plane (defined by the XY-plane of the object space) and the image plane where it is placed. Therefore, we can use the horizon line as the rotational axis of the image plane for image orientation (Rodríguez et al., 2008). Thus, the $(\mathrm{x}, \mathrm{y}, \mathrm{z})$-image coordinate system rotates into the $(\mathrm{X}, \mathrm{Y}, \mathrm{Z})$-object coordinate system through the three following sequential steps.

Step 1: $\vec{v}_{A B}$ is considered as the perpendicular vector to the straight line connecting A and B points in which the second coordinate is positive. We then compute $\psi$ as the angle formed between such a vector and the vertical vector $(0,1)$ - that is the complementary one of the roll angle used in Cornall and Egan (2004). The definition of $\psi$ will change in accordance with the horizon approximation:

Case a) (two points) Being the horizon the straight line defined by the points $\mathrm{A}$ and $\mathrm{B}$ then $\vec{v}_{A B}=$ $\left(y_{a}-y_{b}, x_{b}-x_{a}\right)$, and the angle $\psi$ may be defined as:

$$
\left.\psi=\arctan \left(\left(y_{a}-y_{b}\right) /\left(x_{b}-x_{a}\right)\right) \in\right]-\pi / 2, \pi / 2\left[, \quad \text { being } \mathrm{x}_{\mathrm{a}}<\mathrm{x}_{\mathrm{b}}\right.
$$

Case b) (three points) approaching the horizon through the tangent line to the circumference passing through the points $\mathrm{A}, \mathrm{B}$ and $\mathrm{C}$ then $\vec{v}_{A B}$ is the direction of the line which connects the center of this circumference with the principal point $o=(0,0)$ of the image plane. Consequently, $\vec{v}_{A B}=\left(\mathrm{c}_{\mathrm{x}}, \mathrm{c}_{\mathrm{y}}\right)$ if $\mathrm{c}_{\mathrm{y}}>0$ or $\vec{v}_{A B}=\left(-c_{\mathrm{x}},-c_{\mathrm{y}}\right)$ if $\mathrm{c}_{\mathrm{y}}<0$, where $\left(\mathrm{c}_{\mathrm{x}}, \mathrm{c}_{\mathrm{y}}\right)$ are the coordinates of the circumference center in the image plane with respect to the principal point. This center is previously obtained as the intersection point between the line perpendicular to the segment AC that passes through its midpoint and the line perpendicular to the segment $\mathrm{CB}$ through its midpoint. Finally, the angle $\psi$ is defined as:

$$
\left.\psi=\arctan \left(-\mathrm{c}_{\mathrm{x}} /-\mathrm{c}_{\mathrm{y}}\right)=\arctan \left(\mathrm{c}_{\mathrm{x}} / \mathrm{c}_{\mathrm{y}}\right) \in\right]-\pi / 2, \pi / 2[\text {, if } c y \neq 0
$$


In formula (A.2) it is assumed that the principal point is closer to the horizon than the ABC circumference center. Moreover, $\psi$ has the following definition in the cases in which $\mathrm{c}_{\mathrm{y}}$ could be equal to 0 :

$$
\left\{\begin{array}{l}
\text { if } \mathrm{c}_{\mathrm{y}}=0 \text { and } \mathrm{c}_{\mathrm{x}}<0 \rightarrow \psi=\pi / 2 \\
\text { if } \mathrm{c}_{\mathrm{y}}=0 \text { and } \mathrm{c}_{\mathrm{x}}>0 \rightarrow \psi=-\pi / 2
\end{array}\right.
$$

The xyz-system rotates a clockwise angle $(-\psi)$ in the plane $z=0$, so the new $x$ ' axis will be parallel to the horizon line (see Fig. A.1A). The coordinates of any point in the xyz-system are related with the x'y'z'system by:

$$
\left(\begin{array}{l}
x \\
y \\
z
\end{array}\right)=\left(\begin{array}{ccc}
\cos (-\psi) & -\sin (-\psi) & 0 \\
\sin (-\psi) & \cos (-\psi) & 0 \\
0 & 0 & 1
\end{array}\right)\left(\begin{array}{l}
x^{\prime} \\
y^{\prime} \\
z^{\prime}
\end{array}\right)=\left(\begin{array}{ccc}
\cos (\psi) & \sin (\psi) & 0 \\
-\sin (\psi) & \cos (\psi) & 0 \\
0 & 0 & 1
\end{array}\right)\left(\begin{array}{l}
x^{\prime} \\
y^{\prime} \\
z^{\prime}
\end{array}\right)=R_{\psi}\left(\begin{array}{l}
x^{\prime} \\
y^{\prime} \\
z^{\prime}
\end{array}\right)
$$

In this first step the rotation angle $(-\psi)$ does not consider the position of the horizon line with respect to the X-axis. Formulas (A.1), (A.2) and (A.3) only take into account the orientation of the vector perpendicular to the horizon line with respect to the y-vector direction. For this reason, in the next step we will consider the $\mathrm{d}_{\text {horizon }}$ sign, which represents the minimal distance in the image plane from the principal point to the approached horizon line. Thus, $d_{\text {horizon }}$ could have a negative sign if the closest point of the horizon line to the principal point has a negative y-coordinate. Again, as expected, calculation of $\mathrm{d}_{\text {horizon }}$ depends on the horizon approximation as follows:

Case a) (two points) Following this first approximation, the minimal distance between the principal point and the horizon line is:

$$
\mathrm{d}_{\text {horizon }}=\frac{y_{a} x_{b}-y_{b} x_{a}}{\sqrt{\left(x_{b}-x_{a}\right)^{2}+\left(y_{b}-y_{a}\right)^{2}}}
$$

Case b) (three points) However, in this other case, the minimal distance will be calculated as: $\left|d_{\text {horizon }}\right|=$ $r-\sqrt{c_{x}{ }^{2}+c_{y}{ }^{2}}$, being $r$ the radius of the ABC horizon circumference. It should be remembered that given the characteristics of the horizon, we can assume - without loss of generality - that the principal point is within the circumference. Therefore, the sign of $d_{\text {horizon }}$ is calculated as follows:

$$
\left\{\begin{array}{l}
d_{\text {horizon }}=\mathrm{r}-\sqrt{\mathrm{c}_{\mathrm{x}}{ }^{2}+\mathrm{c}_{\mathrm{y}}^{2}}>0 \text { if } \mathrm{c}_{\mathrm{y}}<0 \\
d_{\text {horizon }}=\sqrt{\mathrm{c}_{\mathrm{x}}{ }^{2}+\mathrm{c}_{\mathrm{y}}{ }^{2}}-r<0 \text { if } \mathrm{c}_{\mathrm{y}}>0
\end{array}\right.
$$

In cases where $\mathrm{c}_{\mathrm{y}}=0$ then:

$$
\left\{\begin{array}{l}
\text { if } \mathrm{c}_{\mathrm{y}}=0 \text { and } \mathrm{c}_{\mathrm{x}}<0 \rightarrow d_{\text {horizon }}=\mathrm{r}-\left|\mathrm{c}_{\mathrm{x}}\right|>0 \\
\text { if } \mathrm{c}_{\mathrm{y}}=0 \text { and } \mathrm{c}_{\mathrm{x}}>0 \rightarrow d_{\text {horizon }}=\left|\mathrm{c}_{\mathrm{x}}\right|-r<0
\end{array}\right.
$$

Moreover, both procedures enable calculating the coordinates of the horizon point $\mathrm{P}\left(\mathrm{x}_{\mathrm{P}}, \mathrm{y}_{\mathrm{P}}\right)$ in the image plane centered at principal point $o=(0,0)$ as:

$$
\left\{\begin{array}{l}
x_{P}=d_{\text {horizon }} * \sin (\psi) \\
y_{P}=d_{\text {horizon }} * \cos (\psi)
\end{array}\right.
$$

It is necessary to consider in (A.8) the appropriate sign of $d_{\text {horizon }}$ as explained above. The observed horizon line could then be indicated in the photos as Fig. A.1A shows. 
Step 2: Once the $\mathrm{x}^{\prime}$ axis is positioned parallel to the horizon line, the image plane must be oriented parallel to the object plane by means of another rotation angle $(-\xi)$ - that is the complementary one of the pitch angle defined in Schwendeman and Thomson (2015). The x'y'z'-coordinate system rotates a clockwise angle $(-\xi)$ to generate an $x$ ' ' $y$ ' $z$ ''-coordinate system, being $x$ ' $=x$ ' and $z$ ' $=\mathrm{Z}$ (the elevation coordinate). Thus, in this step the image orients around the $x^{\prime}$ axis, keeping this parallel to the horizon line and positioning the $y^{\prime}$-axis on a plane which passes through the principal point and is parallel to the object plane (XY) (see Fig. A.2). In this way, $(-\xi)$ coincides with the angle formed between $\mathrm{y}^{*}$ ' axis (whose origin is $C_{c}$ and is parallel to y') and a plane parallel to the terrain pointing to infinity ( $\mathrm{y}^{*}$ '’).

We consider the vector $\vec{v}$ perpendicular to the object plane whose origin is the optical center of the camera. The $\xi$ angle (represented in Fig. A.2) then coincides with the angle formed between such vector $\vec{v}$ and the vector perpendicular to the y'-axis that goes from the camera's centre $C_{c}$ to the principal point 0.

In the triangle formed by $C_{c}$, $o$ and $P$ (the point where the minimum distance between the principal point and the horizon line is reached), the angle $\widehat{C_{c}}$ can be calculated as:

$$
\left.\widehat{C_{c}}=\arctan \left(d_{\text {horizon }} / f\right) \in\right]-\frac{\pi}{2}, \frac{\pi}{2}[
$$

being $f$ the positive value of the camera focal length which coincides with the distance between $C_{c}$ and $o$. We note that the meaning of that angle is from the vector $\overrightarrow{C_{c} O}$ to $\overrightarrow{C_{c} P}$ and its sign coincides with $d_{\text {horizon }}$, distance as defined by formulas (A.5)-(A.7) and which can be positive or negative.

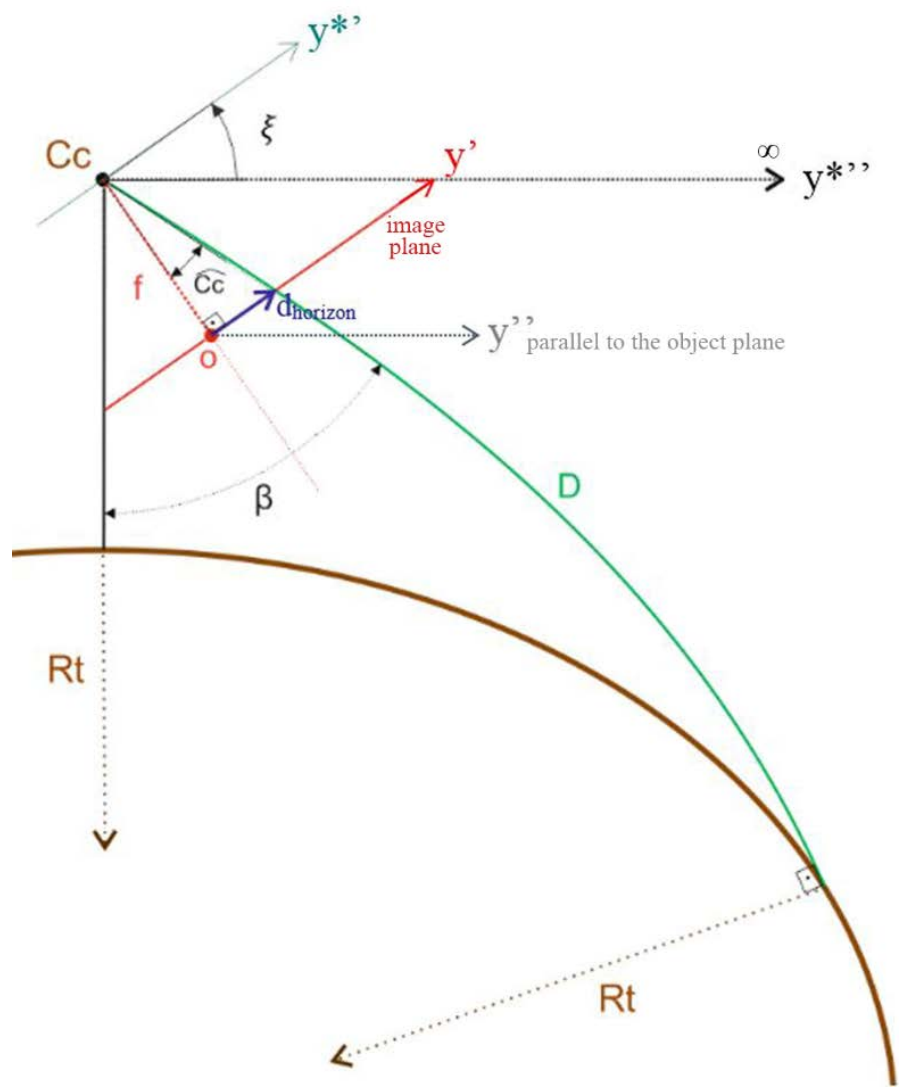

Fig. A.2. Spatial orientation of the image with the graphical definition of angle $\xi$. 
Naturally, the scope of our vision to the horizon will depend on the height $\left(Z_{C_{C}}\right)$ at which the observer is located, as well as the existent geographic features in front of our view. It is known that the field of view of the ground surface extends from the observer's feet to the horizon (Ooi et al., 2001) establishing a trigonometric relationship where an object at infinity is seen as uppermost. Knowing the elevation of the camera $Z_{C_{C}}>0$, and being $R_{t}$ the approximate radius of the Earth $(6371 \mathrm{Km})$, we can then compute an approximation to the geographical distance $\mathrm{D}$ between the observer and the horizon by means of the formula:

$$
D=+\sqrt{\left(Z_{C_{C}}+R_{t}\right)^{2}-R_{t}^{2}}>0
$$

When sitting on the beach, facing the sea, and looking one-meter above the water, the horizon will be distinguished within $\mathrm{D}=3.57 \mathrm{~km}$. However, this observed distance will increase - although disproportionally as height increases.

The next step is to compute the angle $\beta$ between $\vec{v}$ vector and the line between the observer and the horizon. To achieve this we take into account the refraction and terrestrial sphericity correction ( $1 \mathrm{~mm}$ of error in 100 $\mathrm{m}$ of distance). However, sometimes when the observer's elevation is low, this correction becomes irrelevant. The formula to calculate angle $\beta$ is:

$$
\left.\beta=\arccos \left(\frac{z_{C_{C}+0.42 * D^{2} / R_{t}}}{D}\right) \in\right] 0, \pi / 2[
$$

Therefore:

$$
\xi=\beta-\widehat{C_{c}}=\arccos \left(\frac{Z_{C_{C}}+0.42 * \frac{D^{2}}{R_{t}}}{D}\right)-\arctan \left(\frac{d_{\text {horizon }}}{f}\right)
$$

If $\widehat{C_{c}}>0$, $\xi$ definition must be $\xi=\left(\beta-\widehat{C_{c}}\right)>0$, verifying $\left.\xi \in\right] 0, \pi$. The extreme interval values $(\xi=$ $0 \& \xi=\pi$ ) are impossible values because the image plane would be parallel to the object plane - as happens in a vertical photo - where the horizon cannot be seen.

After computing $\xi$ by means of (A.12), the x'y'z'-system rotates the clockwise angle(- $-\xi)$ about the $x^{\prime}$ axis to generate an $x$ ' $y$ '” $z$ '-coordinate system. The coordinates of any point in the $x$ ' $y$ 'z'-system can be calculated from the x'”y'z"'-system by:

$$
\left(\begin{array}{l}
x^{\prime} \\
y^{\prime} \\
z^{\prime}
\end{array}\right)=\left(\begin{array}{ccc}
1 & 0 & 0 \\
0 & \cos (-\xi) & -\sin (-\xi) \\
0 & \sin (-\xi) & \cos (-\xi)
\end{array}\right)\left(\begin{array}{l}
x^{\prime \prime} \\
y^{\prime \prime} \\
z^{\prime \prime}
\end{array}\right)=\left(\begin{array}{ccc}
1 & 0 & 0 \\
0 & \cos (\xi) & \sin (\xi) \\
0 & -\sin (\xi) & \cos (\xi)
\end{array}\right)\left(\begin{array}{l}
x^{\prime \prime} \\
y^{\prime \prime} \\
z^{\prime \prime}
\end{array}\right)=R_{\xi}\left(\begin{array}{l}
x^{\prime \prime} \\
y^{\prime \prime} \\
z^{\prime \prime}
\end{array}\right)
$$

Step 3: The third and last angle $(\lambda)$ of the Euler triad is the azimuth and this positions the coordinate axis ( ${ }^{\prime \prime}$, y', z'”) regarding the real terrain coordinates and around the z"' $=\mathrm{Z}$ axis.

$$
\left(\begin{array}{l}
x^{\prime \prime} \\
y^{\prime \prime} \\
Z^{\prime \prime}
\end{array}\right)=\left(\begin{array}{ccc}
\cos (\lambda) & \sin (\lambda) & 0 \\
-\sin (\lambda) & \cos (\lambda) & 0 \\
0 & 0 & 1
\end{array}\right)\left(\begin{array}{l}
X \\
Y \\
Z
\end{array}\right)=R_{\lambda}\left(\begin{array}{l}
X \\
Y \\
Z
\end{array}\right)
$$


Summarizing, these three new angles are responsible for carrying out the transformation between the image vectors and the terrain vectors with an initial rotation in the image plane around the focal axis, a second rotation turning on the $\mathrm{x}$ ', axis, and the final rotation again around the $\mathrm{Z}$ axis:

$$
\left(\begin{array}{l}
x \\
y \\
Z
\end{array}\right)=R_{\psi} R_{\xi} R_{\lambda}\left(\begin{array}{l}
X \\
Y \\
Z
\end{array}\right)=\left(\begin{array}{ccc}
\cos (\psi) & \sin (\psi) & 0 \\
-\sin (\psi) & \cos (\psi) & 0 \\
0 & 0 & 1
\end{array}\right)\left(\begin{array}{ccc}
1 & 0 & 0 \\
0 & \cos (\xi) & \sin (\xi) \\
0 & -\sin (\xi) & \cos (\xi)
\end{array}\right)\left(\begin{array}{ccc}
\cos (\lambda) & \sin (\lambda) & 0 \\
-\sin (\lambda) & \cos (\lambda) & 0 \\
0 & 0 & 1
\end{array}\right)\left(\begin{array}{l}
X \\
Y \\
Z
\end{array}\right)
$$

Finally, the rotation matrix can produce the change of coordinates since the image to the terrain reference systems is $R_{\lambda \xi \psi}=\left(R_{\psi} R_{\xi} R_{\lambda}\right)^{T}$ :

$$
\left(\begin{array}{l}
X \\
Y \\
Z
\end{array}\right)=\left(\begin{array}{ccc}
\cos \lambda \cos \psi-\sin \lambda \cos \xi \sin \psi & -\sin \lambda \cos \xi \cos \psi-\cos \lambda \sin \psi & \sin \xi \sin \lambda \\
\sin \lambda \cos \psi+\cos \lambda \cos \xi \sin \psi & \cos \lambda \cos \xi \cos \psi-\sin \lambda \sin \psi & -\sin \xi \cos \lambda \\
\sin \xi \sin \psi & \cos \psi \sin \xi & \cos \xi
\end{array}\right)\left(\begin{array}{l}
x \\
y \\
Z
\end{array}\right)
$$

\section{A.2. Obtaining the horizon constraint}

Equating the two rotation matrices $R_{\lambda \xi \psi}$ and $R_{\kappa \varphi \omega}$ it is possible to build the following horizon constraint equations. These will relate $\psi$ and $\xi$ angles - defined by means of (A.1) to (A.3) and (A.12) - with the three known angular EOP, $\{\omega, \varphi, \kappa\}$ :

$$
\begin{aligned}
& \cos (\xi)=\cos (\varphi) \cos (\omega) \\
& \cos (\psi) \sin (\xi)=\cos (\varphi) \sin (\omega) \\
& \sin (\psi) \sin (\xi)=-\sin (\varphi)
\end{aligned}
$$

Because $\psi \in[-\pi / 2, \pi / 2], \xi \in] 0, \pi[$ and $\varphi \in[-\pi / 2, \pi / 2]$ then $\cos (\psi) \geq 0, \sin (\xi)>0$ and $\cos (\varphi) \geq 0$. Consequently, by using equation (A.17) we may conclude that $\sin (\omega) \geq 0$, and so $\omega \in[0, \pi]$. When $\psi \in]-$ $\pi / 2, \pi / 2[$ then $\cos (\psi)>0$ and it is possible to confirm that $\varphi \in]-\pi / 2, \pi / 2[$ and $\omega \in] 0, \pi$ [. Moreover, we notice that $\psi= \pm \pi / 2$ only in cases when we apply equation (A.3) for computing $\psi$. In those cases:

$$
\psi=\frac{\pi}{2} \rightarrow\left\{\begin{array}{cc}
\varphi=-\xi, \omega=0 & \text { if } \xi \in] 0, \frac{\pi}{2}[ \\
\varphi=\xi-\pi, \omega=\pi & \text { if } \xi \in] \frac{\pi}{2}, \pi[ \\
\varphi=-\frac{\pi}{2}, \omega \text { unknown } & \text { if } \xi=\frac{\pi}{2}
\end{array} \text { and } \psi=\frac{-\pi}{2} \rightarrow\left\{\begin{array}{cc}
\varphi=\xi, \omega=0 & \text { if } \xi \in] 0, \frac{\pi}{2}[ \\
\varphi=\xi-\frac{\pi}{2}, \omega=\pi & \text { if } \xi \in] \frac{\pi}{2}, \pi[ \\
\varphi=\frac{\pi}{2}, \omega \text { unknown } & \text { if } \xi=\frac{\pi}{2}
\end{array}\right.\right.
$$

Thus, without loss of generality, in the following steps we will assume that $\psi \in]-\pi / 2, \pi / 2[, \varphi \in]-$ $\pi / 2, \pi / 2[$ and $\omega \in] 0, \pi[$. Equation (A.18) tells us that $\sin (\psi)$ and $\sin (\varphi)$ have different signs and so:

$$
\left.\begin{array}{cc}
\varphi \in] 0, \pi / 2[, & \text { If } \psi \in]-\pi / 2,0[ \\
\varphi \in]-\pi / 2,0[, & \text { If } \psi \in] 0, \pi / 2[ \\
\varphi=0, & \text { If } \psi=0
\end{array}\right\}
$$

However, we can distinguish three different situations in accordance with equation (A.16):

$$
\left.\begin{array}{cc}
\omega \in] 0, \pi / 2[, & \text { If } \xi \in] 0, \pi / 2[ \\
\omega \in] \pi / 2, \pi[, & \text { If } \xi \in] \pi / 2, \pi[ \\
\omega=\pi / 2, & \text { If } \xi=\pi / 2
\end{array}\right\}
$$

Joining (A.17) and (A.18) and considering that $\varphi \in]-\pi / 2, \pi / 2[$ and $\omega \epsilon] 0, \pi[$, equations (A.16)-(A.18) lead to the following equation system: 


$$
\left.\begin{array}{c}
\cos (\xi)=\cos (\varphi) \cos (\omega) \\
\tan (\psi)=\frac{-\sin (\varphi)}{\cos (\varphi) \sin (\omega)}
\end{array}\right\}
$$

1004

1005

1006

1007

1008

1009

1010

1011

1012

1013

1014

1015

1016

1017

1018

1019

1020

1021

1022

1023

1024

1025

1026

1027

1028

1029

1030

1031

1032

1033

1034

1035

1036

being $\cos (\varphi) \sin (\omega)>0$ and $\psi$ and $\xi$ the horizon angles defined by means of (A.1)-(A.3) and (A.12) respectively. Thus, the resulting horizon equations expressed in terms of $\omega$ and $\varphi$ parameters are:

$$
\left\{\begin{array}{c}
\arccos (\cos (\varphi) \cos (\omega))=\xi \epsilon] 0, \pi[\rightarrow \arccos (\cos (\varphi) \cos (\omega))-\xi=0 \\
\left.\arctan \left(\frac{-\sin (\varphi)}{\cos (\varphi) \sin (\omega)}\right)=\psi \epsilon\right]-\pi / 2, \pi / 2\left[\rightarrow \arctan \left(\frac{-\sin (\varphi)}{\cos (\varphi) \sin (\omega)}\right)-\psi=0\right.
\end{array}\right.
$$

\section{A.3. Obtaining from the horizon an initial solution of the camera orientation parameters}

We can use (A.21) to find an initial estimation of the angles $\{\omega, \varphi\}$ that define the orientation of the camera. If we compute the solution of the system (A.21), we obtain:

$$
\sin ^{2}(\varphi)=\frac{1-\cos ^{2}(\xi)}{1+\frac{1}{\tan ^{2}(\psi)}}
$$

Moreover, it is important to avoid numerical errors when $\tan (\psi)$ is close to zero due to $1 / \tan (\psi)^{2} \sim \infty$ so we use the following expression to compute $\varphi$ :

$$
\sin ^{2}(\varphi)=\frac{1-\cos ^{2}(\xi)}{\frac{\tan ^{2}(\psi)+1}{\tan ^{2}(\psi)}}=\frac{\tan ^{2}(\psi) \sin ^{2}(\xi)}{\tan ^{2}(\psi)+1}
$$

Because $0 \leq \sin ^{2}(\xi) \leq 1$, we then confirm that $0 \leq \frac{\tan ^{2}(\psi) \sin ^{2}(\xi)}{\tan ^{2}(\psi)+1} \leq 1$, having finally defined the next mathematical expression for angle $\varphi$ :

$$
\sin (\varphi)= \pm \sqrt{\frac{\tan ^{2}(\psi) \sin ^{2}(\xi)}{\tan ^{2}(\psi)+1}}= \pm \sqrt{\sin ^{2}(\xi) \sin ^{2}(\psi)}= \pm|\sin (\xi) \sin (\psi)|
$$

Focusing on to the first equation of (A.21), the angle $\omega$ can be expressed as:

$$
\cos (\omega)= \pm \sqrt{\frac{\cos ^{2}(\xi)\left(\tan ^{2}(\psi)+1\right)}{1+\cos ^{2}(\xi) \tan ^{2}(\psi)}}= \pm \frac{|\cos (\xi)|}{\sqrt{\cos ^{2}(\psi)+\cos ^{2}(\xi) \sin ^{2}(\psi)}}
$$

Taking into account the relations between $\{\omega, \varphi\}$ and $\{\psi, \xi\}$ proven in (A.19) and (A.20), the next formulas involve all the possible situations in which these two angles can interact in terrestrial photography:

$$
\left\{\begin{array}{c}
\varphi=-\arcsin (\sin (\xi) \sin (\psi)) ; \varphi \epsilon]-\pi / 2, \pi / 2[ \\
\left.\omega=+\arccos \left(\frac{\cos (\xi)}{+\sqrt{\cos ^{2}(\psi)+\cos ^{2}(\xi) \sin ^{2}(\psi)}}\right) ; \omega \epsilon\right] 0, \pi[
\end{array}\right.
$$

Furthermore, regarding the initial value for the third angle $\kappa$, this can be approximated in accordance with the direction in which the photo is pointing. The methodology implemented in C-Pro allows the user to choose the quadrant between cardinal points where the principal camera axis seems to point and associates that with a proposed angular value. Therefore, angle $\kappa$ is initialized as:

$$
\left\{\begin{array}{l}
\text { if photo points between North and East } \rightarrow \kappa=-\pi / 4 ; \\
\text { if photo points between South and East } \rightarrow \kappa=-3 \pi / 4 ; \\
\text { if photo points between North and West } \rightarrow \kappa=\pi / 4 ; \\
\text { if photo points between South and West } \rightarrow \kappa=3 \pi / 4
\end{array}\right.
$$




\section{References}

Aarninkhof, S.G.J., Turner, I.L., Dronkers, T.D.T., Caljouw, M., Nipius, L., 2003. A video-based technique for mapping intertidal beach bathymetry. Coast. Eng. 49, 275-289. doi:10.1016/S0378-3839(03)000644.

Abdel-Aziz, Y.I., Karara, H.M., 1971. Direct linear transformation from comparator coordinates into objects space coordinates in close-range photogrammetry. In Proceedings of the symposium on close-range photogrammetry; pp. 1-18. Urbana, IL: American Society of Photogrammetry.

Almonacid-Caballer, J., Sánchez-García, E., Pardo-Pascual, J.E., Balaguer-Beser, A.A., Palomar-Vázquez, J., 2016. Evaluation of annual mean shoreline position deduced from Landsat imagery as a mid-term coastal evolution indicator. Mar. Geol. 372, 79-88. doi:10.1016/j.margeo.2015.12.015.

Andriolo, U., Sanchez-Garcia, E., Taborda, R., 2016. Using surfcam online streaming images for nearshore hydrodynamics characterization. 4as Jornadas de Engenharia Hidrográfica, Lisbon, Portugal. ISBN. 978-989-705-097-8; pp. 377-380.

Archetti, R., Schiaffi, C.F., Ferrari, M., Brignone, M., Rihouey, D., 2008. Video systems for coastal monitoring. Pranzini, E., Wetzel, L. (Eds.). Beach Eros. Monit. Beachmed-e/OpTIMAL Proj. 101-109.

Bacakoglu, H., Kamel, M.S., 1997. A three-step camera calibration method. IEEE transactions on instrumentation and measurement, Vol. 46, No. 5, 1997, pp. 1165-1172.

Boak, E.H., Turner, I.L., 2005. Shoreline Definition and Detection: A Review. J. Coast. Res. 214, 688-703. doi:10.2112/03-0071.1.

Bouguet, J.Y., 2015. Camera Calibration Toolbox for Matlab. Available at: http:// www.vision.caltech.edu/bouguetj/calib_doc/.

Bracs, M.A., Turner, I.L., Splinter, K.D., Short, A.D., Lane, C., Davidson, M.A., Goodwin, I.D., Pritchard, T., Cameron, D., 2015. Evaluation of Opportunistic Shoreline Monitoring Capability Utilizing Existing “Surfcam” Infrastructure. J. Coast. Res. doi:10.2112/JCOASTRES-D-14-00090.1.

Brignone, M., Schiaffino, C.F., Isla, F.I., Ferrari, M., 2012. A system for beach video-monitoring: Beachkeeper plus. Comput. Geosci. 49, 53-61. doi:10.1016/j.cageo.2012.06.008.

Chandler, J., Fryer, J., Jack, A., 2005. Metric capabilities of low cost digital cameras for close range surface measurement. Photogramm. Rec. 17, 1-16. doi:10.1111/j.1477-9730.2005.00302.x.

Cornall, T.D., Egan, G.K., 2004. Measuring horizon angle from video on a small unmanned air vehicle. In 2nd International Conference on Autonomous Robots and Agents.

Dai, F., Lu, M., Kamat, V.R., 2011. Analytical Approach to Augmenting Site Photos with 3D Graphics of Underground Infrastructure in Construction Engineering Applications. J. Comput. Civ. Eng. 25, 66-74. doi:10.1061/(ASCE)CP.1943-5487.0000072.

Davidson, M., Van Koningsveld, M., de Kruif, A., Rawson, J., Holman, R., Lamberti, A., Medina, R., Kroon, A., Aarninkhof, S., 2007. The CoastView project: Developing video-derived Coastal State Indicators in support of coastal zone management. Coast. Eng. 54, 463-475. doi:10.1016/j.coastaleng.2007.01.007.

Erdman, 1998. Erdman Video System, http://video-monitoring.com. (Accessed 2 March, 2016).

Heikkila, J., Silvén, O., 1997. A four-step camera calibration procedure with implicit image correction. Proc. IEEE Comput. Soc. Conf. Comput. Vis. Pattern Recognit. 1106-1112. doi:10.1109/CVPR.1997.609468.

Holland, K.T., Holman, R. a, Lippmann, T.C., Stanley, J., Plant, N., 1997. Practical Use of Video Imagery in Nearshore Oceanographic Field Studies - Oceanic Engineering, IEEE Journal of 22, 81-92.

Holman, R. a., Stanley, J., 2007. The history and technical capabilities of Argus. Coast. Eng. 54, 477-491. doi:10.1016/j.coastaleng.2007.01.003.

Holman, R., Sallenger, A., Lippmann, T., Haines, J., 1993. The Application of Video Image Processing to the Study of Nearshore Processes. Oceanography 6, 78-85. doi:10.5670/oceanog.1993.02. 
Horus, 2007. Horus System, http://www.horusvideo.com/. (Accessed 5 October, 2016).

Jiménez, J. A., Osorio, A., Marino-Tapia, I., Davidson, M., Medina, R., Kroon, A., Archetti, R., Ciavola, P., Aarnikhof, S.G.J., 2007. Beach recreation planning using video-derived coastal state indicators. Coast. Eng. 54, 507-521. doi:10.1016/j.coastaleng.2007.01.012.

Kim, J., Lee, S., Ahn, H., Seo, D., Seo, D., Lee, J., Choi, C., 2013. Accuracy evaluation of a smartphonebased technology for coastal monitoring. Meas. J. Int. Meas. Confed. 46, 233-248. doi:10.1016/j.measurement.2012.06.010.

Kosta, 2006. KOSTASystem by AZTI, http://www.kostasystem.com/. (Accessed 9 June, 2016).

Ooi, T.L., Wu, B., He, Z.J., 2001. Distance determined by the angular declination below the horizon. Nature 414, 197-200. doi:10.1038/35102562.

Oreifej, O., Lobo, N., Shah, M., 2011. Horizon constraint for unambiguous UAV navigation in planar scenes. Proc. - IEEE Int. Conf. Robot. Autom. 1159-1165. doi:10.1109/ICRA.2011.5979586.

Rodríguez, J., Martín, M.T., Herráez, J., Arias, P., 2008. Three-dimensional image orientation through only one rotation applied to image processing in engineering. Appl. Opt. 47, 6631-6637. doi:10.1364/AO.47.006631.

Sánchez-García, E., Balaguer-Beser, A., Taborda, R., Pardo-Pascual, J.E., 2016. Modelling landscape morphodynamics by terrestrial photogrammetry: An application to beach and fluvial systems. International Archives of Photogrammetry, Remote Sensing and Spatial Information Sciences, 41, 1175-1182. doi:10.5194/isprsarchives-XLI-B8-1175-2016.

Sánchez-García, E., Pardo-Pascual, J.E., Balaguer-Beser, A., Almonacid-Caballer, J., 2015a. Analysis of the shoreline position extracted from Landsat TM and ETM+ imagery. International Archives of Photogrammetry, Remote Sensing and Spatial Information Sciences, 991-998. doi:10.5194/isprsarchives-XL-7-W3-991-2015.

Sánchez-García, E., Pardo-Pascual, J.E., Balaguer-Beser, A., Almonacid-Caballer, J., 2015b. Monitorización de espacios costeros mediante un sistema fotogramétrico: C-Pro. XVI Congr. la Asoc. Española Teledetección “Teledetección Humed. y Espac. protegidos” 281-284.

Schwendeman, M., Thomson, J., 2015. A Horizon-Tracking Method for Shipboard Video Stabilization and Rectification. J. Atmos. Oceanic. Technol., 32, 164-176. doi: 10.1175/JTECH-D-14-00047.1

Seedahmed, G.H., Habib, A.F., 2002. Linear Recovery of the Exterior Orientation Parameters in a Planar Object Space. Int. Arch. Photogramm. Remote Sens. Spat. Inf. Sci. Volume XXX, 245-248.

Taborda, R., Silva, A., 2012. COSMOS: A lightweight coastal video monitoring system. Comput. Geosci. 49, 248-255. doi:10.1016/j.cageo.2012.07.013.

Van Den Heuvel, F.A., 1998. 3D reconstruction from a single image using geometric constraints. ISPRS Journal of Photogrammetry and Remote Sensing, 53, 354-368. doi:10.1016/S0924-2716(98)00019-7. 